\title{
LONG TERM UNDERPERFORMANCE ANOMALY AND ITS DTERMINANT FACTORS ON SEASONED EQUITY OFFERINGS: EVIDENCE FROM TURKEY
}

DOI: 10.17261/Pressacademia.2018.951

JBEF- V.7-ISS.3-2018(2)-p.208-227

Mustafa Cikriikci ${ }^{1}$, Mustafa Ozyesil ${ }^{2}$

${ }^{1}$ Istanbul Aydin University, Istanbul, Turkey. mustafacikrikci@aydin.edu.tr, ORCID: 0000-0002-2805-6079

${ }^{2}$ Istanbul Aydin University, Istanbul, Turkey.

mozyesil@aydin.edu.tr, ORCID: 0000-0002-4442-7087

Date Received: June 30, 2018

Date Accepted: September 18, 2018

To cite this document

Cikrikci, M., Ozyesil, M. (2018). Long term underperformance anomaly and its determinant factors on seasoned equity offerings: evidence from Turkey. Journal of Business, Economic and Finance (JBEF), V.7(3), p.208-227.

Permemant link to this document: http://doi.org/10.17261/Pressacademia.2018.951

Copyright: Published by PressAcademia and limited licenced re-use rights only.

\section{ABSTRACT}

Purpose- This study investigates long term underperformance anomaly existence on Seasoned Equity Offerings (seos) conducted in Borsa Istanbul for the $\mathbf{2 0 1 0}-\mathbf{2 0 1 5}$ period and analyzes its determinant factors.

Methodology- Raw and abnormal returns were calculated then t statistics were obtained for each type of returns. All returns were compared to market average and peer groups returns. The hypotheses were tested via the comparison $t$ statistics and $t$ values. Regression analysis were used to determine what kind of determinants affect long-term price performance. To find out underperformance anomaly's determinants regression analysis was used through Panel Dynamic OLS (PDOLS) method. The analysis was also conducted based on year and sector separately.

Findings- Long-term price performance of firms that performed seos during the 2010-2015 period were calculated lower than market average and peer groups and all findings were statistically significant. Same results were obtained when the analysis was conducted on the basis of year and sector.

Conclusion- Long-term underperformance anomaly was confirmed and it has been determined that Leverage Change Ratio, Private Placemet method and Volume variables have a positive effect on long-term price performance while all other variables have a negative effect.

Keywords: Seasoned equity offering, underperformance, capital increase, abnormal stock returns, price anomalies. JEL Codes: C23, G10, G32

\section{INTRODUCTION}

There are two price anomalies seen in the seasoned equity offerings (seos). These anomalies in the literature are regarded as a short and long term. In short term there is an underpricing anomaly refers that share price performance will be higher than the market average in other words investor can obtain higher return (abnormal return) than the market. In the long term, there is underperformance anomaly suggests that long-term share price performance will be lower than market and peer groups companies that match sample firms by asset size and M/B ratio and don't issue shares during the analysis period.

According to this anomaly, the return of stocks issued offerings shows lower price performance than peer groups and market average in the middle and long-term after the issuance. Based on findings in the literature, most of the studies confirm longterm underperformance anomaly. The period to calculate underperformance anomaly was considered as 3 years in the previous studies. However, some studies have been carried out to investigate the existence of underperformance over a longer period. In some studies, it was researched how long the low performance lasted from the issuance, and it was found that the performance was generally lower around 5-7 years but then it turned to become higher. As in the initial public offerings literature, long-term underperformance anomaly was also seen in seasoned equity offerings (He et al., 2013: 1). 


\section{LITERATURE REVIEW}

Particularly Ritter (1991), and many other studies focused on underperformance anomaly in the literature. Jegadeesh et al. (1993) analyzed 5 year period as a long-term and calculated the average adjusted return - $0.45 \%$ and confirmed underperformance anomaly. Spiess and Affleck (1995) examined 1,247 seos conducted during the period 1975-1985, and confirmed long - term underperformance anomaly. Loughran and Ritter (1997) examined a comprehensive sample of US firms by excluding financial firms and firms that have a lower asset size than 20 Million Usd. during the 1979 - 1989 period and observed underperformance anomaly. Loughran (1997) analyzed 5 year period as a long-term and calculated and decided that there was underperformance anomaly during the analysis period. Teoh et al. (1998) investigated the relationship between earning management practices performed in the financial statements through discretionary accruals and long-term share price performance. They determined that earning management practices causes underperformance in the long-term.

Dubois and Jeanneret (2000) studied on 358 seos and observed underperformance anomaly in Swiss and determined that the underperformance degree was lower than the USA for the analysis period. Eckbo et al. (2000) analyzed more than 7000 seos and confirmed this anomaly. They found that the reason of underperformance anomaly is decreasing the leverage ratio of the issuer firm. Ehrhardt et al. (2000) examined long-term underperformance in 584 seos in Germany during the period of 19601992 and calculated underperformance as - 6\%. Denis and Sarın (2001) indicated that there was an underperformance anomaly and determined this was related to the announcement effect of the financial statements. Clarke et al. (2001) examined longterm stock performance in relation to completed and canceled seos. It was emphasized that insider information trading prior to the public offering for completed seasoned equity offerings is related to long-term stock performance following the completion of public offerings. Islam et al. (2002) pointed out that the firms performed seos in Bangladesh had good business performance before the issuance but a significant decrease was seen in the long term. They also found that decrease ratio is much more severe in smaller firms. Harris (2004) examined 77 seos in the UK during the period 1994-2002 and confirmed underperformance anomaly in the long run. Jeanneret (2005) investigated the long-term underperformance seen in seos in France through offering method company's announcements. In public placement method, there will be serious underperformance in the long run while in private placement he didn't realize any anomalies. Disclosures made by the company management regarding the use of seos revenue reduced underperformance degree.

Bessler and Thies (2006) analyzed the long-term performance of the offerings for the period between 1977 and 1995 in Germany. They found that firms with higher stock performance are much able to go to the public and have a higher chance of success. They stated that the management of the firm especially uses these findings in seos timing. Ngatuni (2007) examined the long-term performance of 818 seos conducted in the UK between 1986 and 1995 and observed seos performed lower in the long term. They found that an investor who received the shares on seos on announcement day would get an average of $41.8 \%$ lower return compared to market and peer groups. Jo and Kim (2008) investigated the relation between ethical behavior and comprehensive public disclosure (material disclosure, financial statement announcements, etc.) and the effect of this relation on seos performances in the long run. They found that comprehensive and accurate information disclosure is positively correlated with post-issue long-term performance. Allen and Soucik (2008) searched the long-term underperformance anomaly in the seos in Australia between 1984 and 1993. They emphasized that the long-term performance of seos will change according to the definition of long-term. They stated that when the long-term is defined as 12 years, long-term underperformance is not valid for seos and also they observed stated that the adjusted returns of stocks will begin to be positive particularly after the 6-7 years.

Virolainen (2009) conducted research on 543 seos in the UK for the period between 1999 and 2007 and found that the seos in the UK are performing poorly in the long run in accordance with the previous literature. But he didn't find underperformance degree calculated as big as in the literature. Bilinski et al. (2012) according to the authors, a seos could improve a firm's stock liquidity and reduce its cost of capital. They were also exploring the role of liquidity risk in explaining the long-term performance of seos. The return of issuer firms was calculated as $46.12 \%$ based on the total of 4,446 seos in the USA for the 5 -year period after the issuance whereas it was $\% 73,79$ for the peer groups firms that match sample firms by M/B ratios. Seal and Matharu (2012) examined long-term performance of seos conducted between the 1999-2005 in Indian based on 5 year analysis period. They confirmed the underperformance anomaly.

Huang et al. (2014) they pointed out that the post-issuance underperformance is more prevalent especially among young issuers, and that this is due to their company risk profile. Gökkaya and Highfield (2014) examined the seos performed in the 1997-2009 period. They found that post-issuance long-term stock performance was negatively correlated with insider stock sales made by senior management (Ceo-Cfo). 


\section{METHODOLOGY}

In order to calculate long-term price performance, 3 years is taken into account as a long-term in line with the previous studies. First, we calculated the raw returns of the stocks as follows :

$\boldsymbol{R}_{i t}=\frac{\left(\boldsymbol{P}_{i t}-\boldsymbol{P}_{i t-1}\right)}{\boldsymbol{P}_{i t-1}}$ (1) $R_{i t}$ : the return of i stock at time $\mathrm{t}, P_{i t}$ : the closing price of the stock $\mathrm{i}$ at time $\mathrm{t}, P_{i t-1}$ : the closing price of the stock $\mathrm{i}$ at the time $\mathrm{t}-1$ (day of effering). In order to calculate abnormal returns for each stocks, market return was calculated the following formula :

$\boldsymbol{R}_{m t}=\frac{\left(\boldsymbol{P}_{m t}-\boldsymbol{P}_{m t-1}\right)}{\boldsymbol{P}_{m t-1}}$ (2) R_mt: The return of the BIST - 100 index at time t, P_mt: The closing price of BIST - 100 index at time t, $P_{-}$(mt-1): The closing price of the BIST - 100 index at time t-1.

Then, the adjusted returns are calculated by subtracting the returns of the BIST 100 National Index from the calculated raw returns.

We assumed that investors followed buy $\&$ hold strategy that means investors purchase stocks from the issuance and hold them during the long term. The abnormal return (AR) of the stocks are calculated by subtracting raw returns obtained in Eq. (1) from the market returns calculated in Eq. (2). AR value can be calculated by the following formula (Asquith and Mullins, 1986: 69):

$\boldsymbol{A} \boldsymbol{R}_{i t}=\boldsymbol{R}_{i t}-\boldsymbol{R}_{\boldsymbol{m t}}$ (3). $A R_{i t}$ : Abnormal return of stock i belonged to firm for at time $\mathrm{t}, R_{i t}:$ The raw return of stock belonged to firm i at time $\mathrm{t}$ and $R_{m t}$; Market return (Bist 100 return) at time $t$.

If day $\mathrm{t}$ which is the first day of the offering is considered as 0 then the average abnormal return of $\mathrm{n}$ shares in any day after the public offering can be calculated via the following formula (Asquith and Mullins, 1986: 69):

$\overline{\boldsymbol{A R}}_{t}=\frac{1}{N} \sum_{i=1}^{n} \boldsymbol{A} \boldsymbol{R}_{i t}$ (4). Another issue that needs to be examined in such analyzes is the calculation of Cumulative Abnormal Return (CAR) and the $t$ statistic for this return which occur between day $t_{1}$ and day $t_{2}$ following offering (Asquith and Mullins, 1986: 69).

$\boldsymbol{C A} \boldsymbol{R}_{t_{1}}^{t_{2}}=\sum_{t=t_{1}}^{t_{2}} \boldsymbol{A} \boldsymbol{R}_{t}$ (5). The average CAR value for each period is calculated using the following formula:

$\overline{C A R}_{t}=\frac{1}{N} \sum_{i=1}^{N} C A R_{i t}(6)$.

The compound abnormal return can be calculated by following Wu and Kwork (2007) as follows:

$\boldsymbol{B} \boldsymbol{H} \boldsymbol{A} \boldsymbol{R}_{\left(T_{1}, T_{2}\right)}=\left[\prod_{t=T_{1}}^{T_{2}}\left(1+R_{i t}\right)\right]-\left[\prod_{t=T_{1}}^{T_{2}}\left(1+R_{m t}\right)\right]$ (7) $R_{i t}:$ the raw return of i stock at time $\mathrm{t}, R_{m t}:$ Market return (Bist 100 return) at time $t$

The average compound abnormal return can be calculated by following Wu and Kwork (2007) as follows:

$\boldsymbol{A} \boldsymbol{v g B H} \boldsymbol{H} \boldsymbol{R}_{\left(\boldsymbol{T}_{1}, \boldsymbol{T}_{2}\right)}=\frac{1}{N} \sum_{i=1}^{N}\left(\left[\prod_{t=T_{1}}^{T_{2}}\left(1+R_{i t}\right)\right]-\left[\prod_{t=T_{1}}^{T_{2}}\left(1+R_{m t}\right)\right]\right)$ (8) $R_{i t}$ : the raw return of i stock at time $\mathrm{t}, R_{m t}:$ Market return (Bist 100 return) at time $t$. The expression $\operatorname{OrtBHAR}_{\left(T_{1}, T_{2}\right)}$ here will be expressed as $\overline{B H A R}$ in terms of ease of use at later stages of the analysis.

In order to test the statistical significance of the average abnormal, cumulative abnormal and compound abnormal returns, by using standard deviations $t$ statistics are calculated first and by comparing the $t$ statistic and critic values obtained from $t$ table following hypotheses were tested:

$H_{0}: \overline{\boldsymbol{A R}}_{\boldsymbol{t}}, \overline{\boldsymbol{C A R}}_{\boldsymbol{t}}, \overline{\boldsymbol{B H A R}}_{\boldsymbol{t}} \leq \mathbf{0}$ Underperformance anomaly is valid according to average returns.

$H_{1}: \overline{\boldsymbol{A R}}_{\boldsymbol{t}}, \overline{\boldsymbol{C A R}}_{\boldsymbol{t}}, \overline{\boldsymbol{B H A R}}_{\boldsymbol{t}}>\mathbf{0}$ Underperformance anomaly is not valid according to average returns.

Hypotheses were tested for each type of returns separately. The t-statistics required to test these hypotheses are calculated using the following formula: (The t-statistics calculated for each type of returns but here AR variable is shown)

$t_{i s t .}=\frac{\overline{A R}_{t}}{\bar{\sigma}\left(A R_{t}\right)} \quad$ (9). Here $\bar{\sigma}\left(A R_{t}\right)$; is the cross-sectional standard deviation at day $\mathrm{t}$, calculated using the following formula:

$$
\bar{\sigma}\left(A R_{t}\right)=\left[\frac{\sum_{i=1}^{n}\left(A R_{i t}-\overline{A R}_{t}\right)^{2}}{n}\right]^{1 / 2}
$$




\section{RESULTS}

\subsection{Stock Price Performance of All Sample for the 36 Months}

Long-term price performance of stocks for whole sample were calculated and findings are represented as follows:

Table 1: Price Performance of the Stocks for the 36 Months

\begin{tabular}{|c|c|c|c|c|c|c|c|c|c|}
\hline Months & $\mathbf{n}$ & $\overline{\bar{R}}$ & t- ist. & $\overline{A R}$ & t- ist. & $\overline{C A R}$ & t- ist. & $\overline{B H A R}$ & t- ist. \\
\hline 1. & 50 & $-2,10$ & $-0,64$ & $-2,95$ & $-0,90$ & $-2,95$ & $-0,90$ & $-2,95$ & $-0,90$ \\
\hline 2. & 50 & 0,23 & 0,08 & $-0,14$ & $-0,05$ & $-3,09$ & $-0,74$ & $-3,60$ & $-0,88$ \\
\hline 3. & 50 & $-0,11$ & $-0,07$ & $-0,77$ & $-0,55$ & $-3,86$ & $-0,88$ & $-4,22$ & $-0,99$ \\
\hline 4. & 50 & 1,03 & 0,44 & $-0,18$ & $-0,08$ & $-4,05$ & $-0,92$ & $-5,51$ & $-1,24$ \\
\hline 5. & 50 & $-2,09$ & $-1,13$ & $-3,09 * *$ & $-1,67$ & $-7,13^{*}$ & $-1,60$ & $-9,20 * *$ & $-2,13$ \\
\hline \multicolumn{10}{|l|}{$\ldots$} \\
\hline 11. & 50 & $-0,86$ & $-0,45$ & $-1,54$ & $-0,96$ & $-12,32 * *$ & $-1,71$ & $-8,91$ & $-0,99$ \\
\hline 12. & 50 & $-0,82$ & $-0,62$ & $-2,41 * * *$ & $-2,38$ & $-14,72 * *$ & $-2,06$ & $-12,19 *$ & $-1,42$ \\
\hline 13. & 50 & $-1,52^{*}$ & $-1,35$ & $-2,22 * * *$ & $-2,45$ & $-16,95 * * *$ & $-2,40$ & $-14,64^{* *}$ & $-1,82$ \\
\hline 14. & 50 & $-2,85^{* *}$ & $-1,67$ & $-1,94$ & $-1,10$ & $-18,89 * * *$ & $-2,77$ & $-16,37^{* *}$ & $-1,80$ \\
\hline 15. & 50 & $-3,63 * * *$ & $-3,48$ & $-2,67 * * *$ & $-3,08$ & $-21,56 * * *$ & $-3,12$ & $-18,03 * *$ & $-2,04$ \\
\hline \multicolumn{10}{|l|}{... } \\
\hline 21. & 50 & $-0,96$ & $-1,03$ & $-1,81^{* *}$ & $-1,80$ & $-28,44 * * *$ & $-3,63$ & $-24,79 * * *$ & $-2,69$ \\
\hline 22. & 50 & $-0,19$ & $-0,13$ & $-2,66 * *$ & $-1,77$ & $-31,10 * * *$ & $-3,94$ & $-29,05 * * *$ & $-3,82$ \\
\hline 23. & 50 & 1,64 & 0,92 & $-0,29$ & $-0,17$ & $-31,39 * * *$ & $-4,12$ & $-32,66 * * *$ & $-5,20$ \\
\hline 24. & 50 & 0,83 & 0,54 & 1,35 & 0,86 & $-30,04 * * *$ & $-3,82$ & $-31,65^{* * *}$ & $-5,01$ \\
\hline 25. & 50 & $-2,18^{*}$ & $-1,42$ & $-2,77^{* *}$ & $-1,97$ & $-32,81 * * *$ & $-4,25$ & $-34,41 * * *$ & $-5,52$ \\
\hline \multicolumn{10}{|l|}{$\ldots$} \\
\hline 31. & 50 & $-1,64$ & $-0,69$ & $-3,10^{*}$ & $-1,40$ & $-40,53 * * *$ & $-4,66$ & $-40,63 * * *$ & $-5,76$ \\
\hline 32. & 50 & $-1,59 *$ & $-1,33$ & $-2,96 * * *$ & $-2,64$ & $-43,49 * * *$ & $-5,02$ & $-43,93 * * *$ & $-6,32$ \\
\hline 33. & 50 & $-0,55$ & $-0,53$ & 0,67 & 0,69 & $-42,82 * * *$ & $-4,94$ & $-43,02 * * *$ & $-6,22$ \\
\hline 34. & 50 & 0,33 & 0,26 & 0,79 & 0,66 & $-42,03 * * *$ & $-4,69$ & $-41,58 * * *$ & $-5,65$ \\
\hline 35. & 50 & $2,31^{*}$ & 1,37 & $2,24^{*}$ & 1,32 & $-39,79 * * *$ & $-4,23$ & $-38,02 * * *$ & $-4,30$ \\
\hline 36. & 50 & 1,11 & 0,81 & 0,22 & 0,15 & $-39,57 * * *$ & $-4,04$ & $-36,14 * * *$ & $-3,64$ \\
\hline
\end{tabular}

Note: $\mathrm{n}$ represents the number of observations. Like Tarı's study (2012: 500), critical values for the t-test were taken as 1,282, 1,645 and 2,326 for the significance levels of $\% 10, \% 5$ and $\% 1$ respectively.

According to the findings in Table 1., the raw returns of stocks that were offered to the public in the following time during the period of 2010-2015 were negative and statistically significant at 13,14, 15, 20, 25 and 32nd months. Positive and statistically significant raw returns were observed at 17 and 35 months. When the abnormal returns are examined, it is seen that the abnormal returns are negative and statistically significant at 5, 12, 13, 15, 20, 21, 22, 25, 26th months and it appears that it provides a positive and statistically significant abnormal return at 35th month. The average cumulative abnormal returns and average compound returns continuously cause losing for the investors. It is seen that an investor who took these shares and held them for 36 months lost between $36.14 \%$ and $39.57 \%$ on average, which is also statistically significant. Based on this finding, the $\mathrm{HO}$ hypothesis can be accepted and it can be concluded that underperformance is valid for stocks that are offered in the period 2010-2015.

\subsection{Stock Price Performance Based on Year and Sector}

The first three-year stock price performance has also been analyzed on a yearly basis as follows:

Table 2: Price Performance of Shares Issued in 2010 During First 36 Months After Offerings

\begin{tabular}{l|llllllllll}
\hline \multicolumn{1}{l}{ Months } & $\mathbf{n}$ & $\bar{R}$ & t- ist. & \multicolumn{1}{c}{$\overline{A R}$} & t- ist. & $\overline{C A R}$ & t- ist. & $\overline{B H A R}$ & t- ist. \\
\hline 1. & 9 & $-6,86^{* *}$ & $-2,20$ & $-11,37^{* * *}$ & $-2,99$ & $-11,37^{* * *}$ & $-2,99$ & $-11,37^{* * *}$ & $-2,99$ \\
2. & 9 & 13,15 & 1,04 & 11,21 & 0,94 & $-0,16$ & $-0,01$ & 0,19 & 0,01 \\
3. & 9 & 2,13 & 0,78 & 0,84 & 0,34 & 0,69 & 0,05 & 2,80 & 0,18 \\
4. & 9 & $7,35^{*}$ & 1,37 & 5,91 & 1,18 & 6,60 & 0,54 & 5,98 & 0,46 \\
5. & 9 & $-6,07^{*}$ & $-1,52$ & $-6,04^{*}$ & $-1,35$ & 0,56 & 0,05 & $-3,25$ & $-0,32$ \\
.. & & & & & & & & &
\end{tabular}




\begin{tabular}{|c|c|c|c|c|c|c|c|c|c|}
\hline 11. & 9 & $-8,88 * *$ & $-2,13$ & $-4,96 * *$ & $-1,81$ & 2,32 & 0,13 & 8,67 & 0,39 \\
\hline 12. & 9 & $-0,98$ & $-0,18$ & $-1,25$ & $-0,28$ & 1,08 & 0,06 & 1,73 & 0,11 \\
\hline 13. & 9 & $-3,48^{*}$ & $-1,37$ & $-1,15$ & $-0,76$ & $-0,08$ & 0,00 & $-2,48$ & $-0,18$ \\
\hline 14. & 9 & $-6,83$ & $-0,97$ & $-3,20$ & $-0,45$ & $-3,28$ & $-0,27$ & $-13,15^{* *}$ & $-1,96$ \\
\hline 15. & 9 & $-7,24 * * *$ & $-2,33$ & $-5,05 * * *$ & $-3,04$ & $-8,33$ & $-0,69$ & $-16,25^{* *}$ & $-2,26$ \\
\hline$\ldots$ & & & & & & & & & \\
\hline 21. & 9 & $-2,20$ & $-0,85$ & $-4,81^{* * *}$ & $-2,96$ & $-20,44^{*}$ & $-1,53$ & $-27,42^{* * *}$ & $-2,97$ \\
\hline 22. & 9 & 1,14 & 0,42 & $-2,77^{*}$ & $-1,55$ & $-23,20 * *$ & $-1,72$ & $-31,40 * * *$ & $-3,39$ \\
\hline 23. & 9 & $-2,24$ & $-0,63$ & $-4,50^{*}$ & $-1,30$ & $-27,70 * * *$ & $-2,46$ & $-37,31 * * *$ & $-5,72$ \\
\hline 24. & 9 & 3,25 & 0,49 & 1,01 & 0,13 & $-26,69 * *$ & $-1,76$ & $-37,60 * * *$ & $-5,20$ \\
\hline 25. & 9 & $-2,04$ & $-0,89$ & $-4,56 * *$ & $-1,82$ & $-31,24 * *$ & $-1,92$ & $-41,38 * * *$ & $-5,85$ \\
\hline$\cdots$ & & & & & & & & & \\
\hline 31. & 9 & 0,17 & 0,05 & $-3,27$ & $-0,98$ & $-52,24 * * *$ & $-3,35$ & $-65,41 * * *$ & $-6,03$ \\
\hline 32. & 9 & 1,39 & 0,70 & $-1,10$ & $-0,62$ & $-53,35 * * *$ & $-3,28$ & $-68,13 * * *$ & $-5,57$ \\
\hline 33. & 9 & $-4,19 *$ & $-1,62$ & $-0,65$ & $-0,36$ & $-53,99 * * *$ & $-3,59$ & $-66,09 * * *$ & $-6,13$ \\
\hline 34. & 9 & $-3,79 * * *$ & $-3,01$ & $-1,88^{*}$ & $-1,32$ & $-55,88 * * *$ & $-3,88$ & $-65,54 * * *$ & $-6,62$ \\
\hline 35. & 9 & $-1,01$ & $-0,76$ & 0,41 & 0,18 & $-55,46 * * *$ & $-4,10$ & $-64,91 * * *$ & $-8,50$ \\
\hline 36. & 9 & 2,11 & 0,80 & 2,31 & 0,69 & $-53,15 * * *$ & $-3,56$ & $-62,90 * * *$ & $-6,25$ \\
\hline
\end{tabular}

Note: $\mathrm{n}$ represents the number of observations. Like Tarı's study (2012: 500), critical values for the t-test were taken as 1,282, 1,645 and 2,326 for the significance levels of $\% 10, \% 5$ and $\% 1$ respectively.

According to the findings in shown Table 2, the average raw returns of stocks that were offered to the public for 2010 were negative and statistically significant in the first month, 5th, 11th, 13th, 15th, 20th, 33th and 34th months from the day when they started to trade in the stock market. Positive and statistically significant values were found in the month 4th, 7th and 28th months. Average abnormal returns were found to be positive and statistically significant at 7th month. By the way, AR, CAR and BHAR values were observed as negative and statistically significant in the first month and most of the other months. Given the fact that average cumulative abnormal returns and average compound abnormal returns have consistently negative and statistically significant values since the 14th month, it has been decided that the hypothesis $\mathrm{HO}$ can be accepted and the underperformance for stocks that are offered to the public in 2010 are valid. It has been determined that investors who purchased these stocks and hold them for 36 months will loss around \%53 - \%63.

Table 3: Price Performance of Shares Issued in 2011 During First 36 Months After Offerings

\begin{tabular}{|c|c|c|c|c|c|c|c|c|c|}
\hline Months & $\mathbf{n}$ & $\bar{R}$ & t- ist. & $\overline{A R}$ & t- ist. & $\overline{C A R}$ & t- ist. & $\overline{B H A R}$ & t- ist. \\
\hline 1. & 15 & 4,96 & 0,60 & 5,81 & 0,76 & 5,81 & 0,76 & 5,81 & 0,76 \\
\hline 2. & 15 & $-10,91 * * *$ & $-3,37$ & $-9,34^{*}$ & $-2,95$ & $-3,52$ & $-0,67$ & $-6,85 * * *$ & $-2,55$ \\
\hline 3. & 15 & 1,06 & 0,39 & 0,79 & 0,40 & $-2,73$ & $-0,50$ & $-6,25 * *$ & $-1,87$ \\
\hline 4. & 15 & $-3,95^{*}$ & $-1,59$ & $-1,64$ & $-0,81$ & $-4,38$ & $-0,86$ & $-7,83 * * *$ & $-2,56$ \\
\hline 5. & 15 & 0,19 & 0,06 & 0,44 & 0,17 & $-3,94$ & $-0,77$ & $-8,00 * * *$ & $-2,37$ \\
\hline \multicolumn{10}{|l|}{... } \\
\hline 11. & 15 & 4,03 & 0,89 & 2,55 & 0,67 & $-5,17$ & $-0,56$ & $-12,67^{* *}$ & $-1,74$ \\
\hline 12. & 15 & 0,35 & 0,18 & $-2,94 * *$ & $-1,98$ & $-8,11$ & $-0,91$ & $-16,12^{* *}$ & $-2,18$ \\
\hline 13. & 15 & 0,53 & 0,29 & $-3,15^{*}$ & $-1,61$ & $-11,26$ & $-1,26$ & $-20,05 * * *$ & $-2,76$ \\
\hline 14. & 15 & $-5,34 * * *$ & $-3,07$ & $-7,19 * * *$ & $-4,78$ & $-18,46^{* *}$ & $-2,00$ & $-26,70 * * *$ & $-3,62$ \\
\hline 15. & 15 & $-3,61 * *$ & $-1,91$ & $-5,84 * * *$ & $-4,44$ & $-24,30 * * *$ & $-2,69$ & $-31,73 * * *$ & $-4,48$ \\
\hline \multicolumn{10}{|l|}{$\ldots$} \\
\hline 21. & 15 & 0,88 & 0,39 & $-0,07$ & $-0,03$ & $-42,57 * * *$ & $-3,83$ & $-45,90 * * *$ & $-4,92$ \\
\hline 22. & 15 & $-3,09 *$ & $-1,36$ & $-4,10^{*}$ & $-1,51$ & $-46,67 * * *$ & $-3,92$ & $-47,93 * * *$ & $-4,70$ \\
\hline 23. & 15 & 0,09 & 0,05 & $-2,82^{*}$ & $-1,38$ & $-49,49 * * *$ & $-4,04$ & $-51,10 * * *$ & $-4,73$ \\
\hline 24. & 15 & $-0,24$ & $-0,11$ & 1,00 & 0,55 & $-48,50 * * *$ & $-4,01$ & $-49,15^{* * *}$ & $-4,57$ \\
\hline 25. & 15 & $-3,81 * *$ & $-1,68$ & $-3,69 * *$ & $-1,83$ & $-52,19 * * *$ & $-4,57$ & $-52,60 * * *$ & $-5,45$ \\
\hline \multicolumn{10}{|l|}{$\ldots$} \\
\hline 31. & 15 & $-3,32 *$ & $-1,64$ & $-3,89 * * *$ & $-2,50$ & $-55,57 * * *$ & $-4,21$ & $-49,86 * * *$ & $-4,50$ \\
\hline 32. & 15 & $-3,13$ & $-1,07$ & $-3,83^{*}$ & $-1,58$ & $-59,41 * * *$ & $-4,30$ & $-52,36 * * *$ & $-4,56$ \\
\hline 33. & 15 & 0,91 & 0,36 & 1,19 & 0,46 & $-58,21 * * *$ & $-4,19$ & $-52,11 * * *$ & $-4,45$ \\
\hline 34. & 15 & 0,54 & 0,21 & 0,61 & 0,26 & $-57,61 * * *$ & $-4,14$ & $-52,50 * * *$ & $-4,44$ \\
\hline 35. & 15 & 0,46 & 0,32 & 0,39 & 0,22 & $-57,22 * * *$ & $-3,94$ & $-52,32 * * *$ & $-4,43$ \\
\hline 36. & 15 & 1,19 & 0,59 & $-0,35$ & $-0,18$ & $-57,58 * * *$ & $-3,96$ & $-52,85 * * *$ & $-4,39$ \\
\hline
\end{tabular}


Note: $\mathrm{n}$ represents the number of observations. Like Tarı's study (2012: 500), critical values for the t-test were taken as 1,282, 1,645 and 2,326 for the significance levels of $\% 10, \% 5$ and $\% 1$ respectively.

According to the findings represented in Table 3; it is seen that the average compound return of stocks that were offered to the public in 2011 was negative and statistically significant. In this case, the HO hypothesis was accepted and it was decided that underperformance would be valid. It has been seen that an investor who holds these shares for 36 months since the issuance date will suffer an average loss of more than $50 \%$.

Table 4: Price Performance of Shares Issued in 2012 During First 36 Months After Offerings

\begin{tabular}{|c|c|c|c|c|c|c|c|c|c|}
\hline Months & 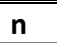 & $\bar{R}$ & t- ist. & $\overline{A R}$ & t- ist. & $\overline{C A R}$ & t- ist. & $\overline{B H A R}$ & t- ist. \\
\hline 1. & 11 & $-7,87 * *$ & $-1,87$ & $-12,21 * * *$ & $-3,15$ & $-12,21 * * *$ & $-3,15$ & $-12,21 * * *$ & $-3,15$ \\
\hline 2. & 11 & 1,38 & 0,53 & $-2,16$ & $-0,72$ & $-14,37^{* * *}$ & $-2,39$ & $-14,21 * * *$ & $-2,56$ \\
\hline 3. & 11 & $-3,00 * *$ & $-1,74$ & $-5,84 * * *$ & $-3,62$ & $-20,21 * * *$ & $-3,04$ & $-19,87 * * *$ & $-3,33$ \\
\hline 4. & 11 & $-2,87^{*}$ & $-1,63$ & $-6,69 * * *$ & $-3,73$ & $-26,90 * * *$ & $-3,48$ & $-26,12 * * *$ & $-3,86$ \\
\hline 5. & 11 & $-2,21$ & $-0,52$ & $-5,36^{*}$ & $-1,35$ & $-32,26 * * *$ & $-3,54$ & $-31,25 * * *$ & $-3,97$ \\
\hline$\ldots$ & & & & & & & & & \\
\hline 11. & 11 & $-5,17^{*}$ & $-1,44$ & $-6,71 * *$ & $-2,05$ & $-52,60 * * *$ & $-3,49$ & $-43,12 * * *$ & $-3,24$ \\
\hline 12. & 11 & $-3,20$ & $-1,04$ & $-3,20$ & $-1,04$ & $-54,11 * * *$ & $-3,46$ & $-44,13 * * *$ & $-3,53$ \\
\hline 13. & 11 & $-4,77^{*}$ & $-1,62$ & $-0,53$ & $-0,35$ & $-54,64 * * *$ & $-3,40$ & $-41,45 * * *$ & $-3,30$ \\
\hline 14. & 11 & 1,64 & 0,43 & 3,95 & 1,05 & $-50,69 * * *$ & $-3,33$ & $-38,22 * * *$ & $-2,87$ \\
\hline 15. & 11 & $-2,96$ & $-1,26$ & 0,69 & 0,33 & $-49,99 * * *$ & $-3,14$ & $-35,62 * * *$ & $-2,65$ \\
\hline$\ldots$ & & & & & & & & & \\
\hline 21. & 11 & $-1,92 * * *$ & $-2,48$ & $-1,65^{* *}$ & $-2,13$ & $-56,78 * * *$ & $-3,17$ & $-41,80 * * *$ & $-3,12$ \\
\hline 22. & 11 & $-0,76$ & $-0,46$ & $-2,61 * *$ & $-2,01$ & $-59,39 * * *$ & $-3,18$ & $-42,97 * * *$ & $-2,97$ \\
\hline 23. & 11 & $7,28 * *$ & 1,83 & $4,36^{*}$ & 1,38 & $-55,03 * * *$ & $-3,03$ & $-42,14 * * *$ & $-2,73$ \\
\hline 24. & 11 & 1,63 & 0,88 & $-1,10$ & $-0,66$ & $-56,13 * * *$ & $-3,11$ & $-44,58 * * *$ & $-2,92$ \\
\hline 25. & 11 & $4,62 * *$ & 2,31 & 1,17 & 0,74 & $-54,96 * * *$ & $-2,84$ & $-42,67 * * *$ & $-2,48$ \\
\hline$\ldots$ & & & & & & & & & \\
\hline 31. & 11 & $-2,99 *$ & $-1,32$ & $-3,50 * *$ & $-1,72$ & $-59,01 * * *$ & $-2,32$ & $-39,02 * *$ & $-1,77$ \\
\hline 32. & 11 & $-0,95$ & $-0,65$ & $-1,30$ & $-0,73$ & $-60,31 * * *$ & $-2,37$ & $-40,95 * *$ & $-1,86$ \\
\hline 33. & 11 & $-2,19 * *$ & $-1,69$ & 0,71 & 0,43 & $-59,60 * * *$ & $-2,39$ & $-39,37 * *$ & $-1,80$ \\
\hline 34. & 11 & $-3,14 * *$ & $-1,87$ & $-2,13^{*}$ & $-1,32$ & $-61,72 * * *$ & $-2,48$ & $-40,11 * *$ & $-1,80$ \\
\hline 35. & 11 & $5,32 *$ & 1,55 & 4,14 & 1,21 & $-57,58 * *$ & $-2,29$ & $-34,67^{*}$ & $-1,31$ \\
\hline 36. & 11 & $-2,07$ & $-0,45$ & $-0,58$ & $-0,12$ & $-58,16 * *$ & $-2,17$ & $-30,22$ & $-1,02$ \\
\hline
\end{tabular}

Note: $\mathrm{n}$ represents the number of observations. Like Tarı's study (2012: 500), critical values for the t-test were taken as 1,282, 1,645 and 2,326 for the significance levels of $\% 10, \% 5$ and $\% 1$ respectively.

According to the findings in tables 4 , it is seen that the average cumulative abnormal returns and the average compound abnormal returns (excluding the last month) of stocks that were offered in 2012 were negative and statistically significant. In this case, the $\mathrm{HO}$ hypothesis was accepted and it was decided that underperformance for offerings performed in 2012 is valid. Although it is considered positive and statistically significant at 23, 25 and 35 months according to the average raw yields, it is seen that an investor who took these shares on the date of the public offering and held them for 36 months lost $30 \%-58 \%$.

Table 5: Price Performance of Shares Issued in 2013 During First 36 Months After Offerings

\begin{tabular}{l|lllllllll}
\hline \hline Months & $\mathbf{n}$ & \multicolumn{1}{c}{$\bar{R}$} & t- ist. & \multicolumn{1}{c}{$\overline{A R}$} & t- ist. & $\overline{C A R}$ & t- ist. & $\overline{B H A R}$ & t- ist. \\
\hline $\mathbf{1 .}$ & 11 & $-6,20^{*}$ & $-1,59$ & $-2,27$ & $-0,56$ & $-2,27$ & $-0,56$ & $-2,27$ & $-0,56$ \\
$\mathbf{2 .}$ & 11 & 1,52 & 0,38 & 3,29 & 0,63 & 1,02 & 0,14 & 1,25 & 0,17 \\
$\mathbf{3 .}$ & 11 & 0,54 & 0,11 & 2,83 & 0,61 & 3,85 & 0,43 & 5,03 & 0,55 \\
$\mathbf{4 .}$ & 11 & $13,97^{* * *}$ & 3,36 & $10,34^{* *}$ & 2,01 & $14,19^{*}$ & 1,40 & $15,96 *$ & 1,38 \\
$\mathbf{5 .}$ & 11 & $-0,55$ & $-0,13$ & $-1,72$ & $-0,36$ & $12,47^{*}$ & 1,30 & 13,34 & 1,23 \\
$\mathbf{\ldots}$ & & & & & & & & & \\
$\mathbf{1 1 .}$ & 11 & $4,31^{* *}$ & 1,78 & 1,83 & 0,74 & 13,78 & 0,87 & 27,46 & 0,94 \\
$\mathbf{1 2}$ & 11 & 0,42 & 0,43 & $-3,24^{* * *}$ & $-3,03$ & 10,53 & 0,65 & 24,81 & 0,83 \\
$\mathbf{1 3 .}$ & 11 & $-0,47$ & $-0,22$ & $-5,34^{* * *}$ & $-3,56$ & 5,19 & 0,32 & 18,15 & 0,64 \\
$\mathbf{1 4 .}$ & 11 & 0,32 & 0,13 & 0,20 & 0,07 & 5,39 & 0,30 & 24,39 & 0,69
\end{tabular}




\begin{tabular}{l|lllllllll} 
15. & 11 & $-1,58$ & $-0,84$ & $-0,26$ & $-0,14$ & 5,13 & 0,28 & 23,47 & 0,69 \\
.. & & & & & & & & & \\
21. & 11 & $-2,59 * *$ & $-1,69$ & $-1,82$ & $-0,82$ & 11,44 & 0,58 & 26,92 & 0,81 \\
22. & 11 & $-2,19$ & $-0,71$ & $-5,13^{*}$ & $-1,50$ & 6,31 & 0,35 & 14,00 & 0,60 \\
23. & 11 & $-0,38$ & $-0,07$ & $-0,46$ & $-0,08$ & 5,85 & 0,37 & 4,45 & 0,34 \\
24. & 11 & $-1,83$ & $-1,01$ & $2,80^{*}$ & 1,64 & 8,65 & 0,55 & 6,63 & 0,52 \\
25. & 11 & $-6,62^{*}$ & $-1,64$ & $-4,53$ & $-1,16$ & 4,12 & 0,29 & $-1,14$ & $-0,10$ \\
$\mathbf{\ldots}$ & & & & & & & & & \\
31. & 11 & $-6,27^{*}$ & $-1,41$ & $-8,43^{* *}$ & $-2,17$ & $-2,58$ & $-0,18$ & $-9,57$ & $-0,81$ \\
32. & 11 & $-3,68^{*}$ & $-1,41$ & $-5,03^{* *}$ & $-1,65$ & $-7,61$ & $-0,61$ & $-15,49 *$ & $-1,59$ \\
33. & 11 & 0,55 & 0,35 & 1,51 & 1,12 & $-6,10$ & $-0,46$ & $-13,92^{*}$ & $-1,41$ \\
34. & 11 & $6,54^{* *}$ & 2,04 & $6,78^{* *}$ & 2,00 & 0,69 & 0,04 & $-5,64$ & $-0,42$ \\
35. & 11 & 6,76 & 1,09 & $7,46^{*}$ & 1,31 & 8,15 & 0,41 & 6,48 & 0,31 \\
36. & 11 & $3,91^{* *}$ & 1,71 & 1,66 & 0,68 & 9,81 & 0,47 & 11,47 & 0,46 \\
\hline
\end{tabular}

Note: $\mathrm{n}$ represents the number of observations. Like Tarı's study (2012: 500), critical values for the t-test were taken as 1,282, 1,645 and 2,326 for the significance levels of $\% 10, \% 5$ and $\% 1$ respectively.

According to the findings shown in Table 5, positive and statistically significant results were observed at 4th, 11th, 16th, 28th, 34 th and 36th months although negative results were seen in the first months in 2013 . Therefore, it can be concluded that the $\mathrm{HO}$ hypothesis is rejected and over performance for stocks that are offered to the public for 2013 is valid.

Table 6: Price Performance of Shares Issued in 2014 During First 36 Months After Offerings

\begin{tabular}{|c|c|c|c|c|c|c|c|c|c|}
\hline Months & n & $\overline{\bar{R}}$ & t- ist. & $\overline{\overline{A R}}$ & t- ist. & $\overline{\overline{C A R}}$ & t- ist. & $\overline{\overline{B H A R}}$ & t- ist. \\
\hline 1. & 4 & 9,22 & 0,41 & 6,69 & 0,29 & 6,69 & 0,29 & 6,69 & 0,29 \\
\hline 2. & 4 & 6,27 & 1,08 & 4,92 & 0,76 & 11,61 & 0,39 & 15,93 & 0,50 \\
\hline 3. & 4 & $-3,35$ & $-0,69$ & $-6,22 *$ & $-1,44$ & 5,39 & 0,21 & 5,15 & 0,21 \\
\hline 4. & 4 & $-19,42$ & $-1,22$ & $-19,47^{*}$ & $-1,29$ & $-14,08$ & $-0,99$ & $-25,06 * *$ & $-1,92$ \\
\hline 5. & 4 & $-5,66$ & $-0,75$ & $-7,17$ & $-0,83$ & $-21,24 *$ & $-1,53$ & $-28,43 * *$ & $-1,69$ \\
\hline \multicolumn{10}{|l|}{$\ldots$} \\
\hline 11. & 4 & $-3,48 * * *$ & $-2,41$ & $-4,25 * * *$ & $-2,36$ & $-33,03 * *$ & $-2,20$ & $-40,37 * * *$ & $-2,63$ \\
\hline 12. & 4 & $-1,67$ & $-0,70$ & $-3,18 * * *$ & $-2,58$ & $-36,21 * * *$ & $-2,42$ & $-42,73 * * *$ & $-2,93$ \\
\hline 13. & 4 & 1,27 & 0,27 & 2,78 & 0,56 & $-33,44 * *$ & $-1,90$ & $-38,09 * * *$ & $-2,32$ \\
\hline 14. & 4 & $-5,61 * *$ & $-2,07$ & $-1,54$ & $-0,58$ & $-34,98 * *$ & $-1,88$ & $-36,81 * *$ & $-2,15$ \\
\hline 15. & 4 & $-3,10^{*}$ & $-1,45$ & $-1,32$ & $-1,23$ & $-36,30 * *$ & $-1,91$ & $-36,37 * *$ & $-2,31$ \\
\hline \multicolumn{10}{|l|}{$\ldots$} \\
\hline 21. & 4 & 2,02 & 0,53 & $-1,98$ & $-0,78$ & $-25,22^{*}$ & $-1,48$ & $-35,06 * * *$ & $-2,66$ \\
\hline 22. & 4 & 14,71 & 1,26 & 9,70 & 0,86 & $-15,52$ & $-0,64$ & $-33,02 * * *$ & $-2,48$ \\
\hline 23. & 4 & $6,20 * *$ & 2,26 & $6,35^{*}$ & 1,64 & $-9,17$ & $-0,34$ & $-29,00 * *$ & $-1,93$ \\
\hline 24. & 4 & 4,52 & 0,68 & $6,16^{*}$ & 1,50 & $-3,00$ & $-0,11$ & $-22,39 *$ & $-1,39$ \\
\hline 25. & 4 & $-2,91$ & $-0,28$ & $-1,34$ & $-0,13$ & $-4,35$ & $-0,18$ & $-19,33$ & $-0,85$ \\
\hline \multicolumn{10}{|l|}{... } \\
\hline 31. & 4 & 17,05 & 0,67 & 16,06 & 0,68 & $-11,38$ & $-0,38$ & $-40,04 * * *$ & $-5,07$ \\
\hline 32. & 4 & 1,44 & 0,32 & $-2,69$ & $-0,88$ & $-14,07$ & $-0,46$ & $-44,23 * * *$ & $-5,73$ \\
\hline 33. & 4 & $3,66^{*}$ & 1,36 & $-0,75$ & $-0,23$ & $-14,82$ & $-0,44$ & $-47,01 * * *$ & $-5,39$ \\
\hline 34. & 4 & 1,25 & 0,25 & $-0,94$ & $-0,34$ & $-15,76$ & $-0,48$ & $-49,56 * * *$ & $-6,68$ \\
\hline 35. & 4 & $-3,78$ & $-0,69$ & $-6,29$ & $-1,10$ & $-22,05$ & $-0,74$ & $-55,53 * * *$ & $-8,83$ \\
\hline 36. & 4 & $-0,40$ & $-0,19$ & $-4,11 * * *$ & $-3,73$ & $-26,16$ & $-0,91$ & $-60,48 * * *$ & $-9,64$ \\
\hline
\end{tabular}

Note: $\mathrm{n}$ represents the number of observations. Like Tarı's study (2012: 500), critical values for the t-test were taken as 1,282, 1,645 and 2,326 for the significance levels of $\% 10, \% 5$ and $\% 1$ respectively.

According to the findings represented in Table 6, while the average returns of the stocks that were offered in 2014 were positive and statistically significant at the 23rd and 33rd months, the average cumulative abnormal returns and the average compound abnormal returns were found to be generally negative and statistically significant. HO hypothesis was accepted for this reason and it was decided that underperformance would be valid for stocks that were offered to the public in 2014. It has been seen that an investor who holds these shares for 36 months will suffer an average loss of $60.48 \%$. 
The first three-year stock price performance has also been analyzed on a sector basis as follows:

Table 7: Price Performance of Industrial Firms' Shares for the first 36 Months

\begin{tabular}{|c|c|c|c|c|c|c|c|c|c|}
\hline Months & $\mathbf{n}$ & $\overline{\bar{R}}$ & t- ist. & $\overline{\overline{A R}}$ & t- ist. & $\overline{\overline{C A R}}$ & t- ist. & $\overline{\overline{B H A R}}$ & t- ist. \\
\hline 1. & 22 & 3,14 & 0,48 & 1,13 & 0,18 & 1,13 & 0,18 & 1,13 & 0,18 \\
\hline 2. & 22 & $7,58^{*}$ & 1,28 & $7,32 *$ & 1,27 & 8,45 & 1,07 & 6,88 & 0,88 \\
\hline 3. & 22 & 0,99 & 0,32 & 0,01 & 0,00 & 8,46 & 1,05 & 6,60 & 0,81 \\
\hline 4. & 22 & 1,25 & 0,28 & $-0,52$ & $-0,13$ & 7,95 & 1,07 & 4,10 & 0,49 \\
\hline 5. & 22 & $-2,74$ & $-0,86$ & $-5,07^{*}$ & $-1,56$ & 2,88 & 0,43 & $-2,87$ & $-0,40$ \\
\hline \multicolumn{10}{|l|}{... } \\
\hline 11. & 22 & 1,62 & 0,45 & $-0,40$ & $-0,13$ & 1,19 & 0,10 & 6,19 & 0,34 \\
\hline 12. & 22 & $-3,31 *$ & $-1,54$ & $-3,64 * * *$ & $-2,35$ & $-2,45$ & $-0,20$ & 0,98 & 0,06 \\
\hline 13. & 22 & $-0,91$ & $-0,54$ & $-1,94^{*}$ & $-1,54$ & $-4,39$ & $-0,36$ & $-2,84$ & $-0,17$ \\
\hline 14. & 22 & $-4,11 *$ & $-1,31$ & $-3,96 *$ & $-1,27$ & $-8,35$ & $-0,69$ & $-6,60$ & $-0,34$ \\
\hline 15. & 22 & $-4,37 * * *$ & $-2,43$ & $-2,29 * *$ & $-1,83$ & $-10,64$ & $-0,87$ & $-7,96$ & $-0,42$ \\
\hline \multicolumn{10}{|l|}{$\ldots$} \\
\hline 21. & 22 & 0,46 & 0,32 & $-0,61$ & $-0,38$ & $-14,59$ & $-1,09$ & $-15,60$ & $-0,81$ \\
\hline 22. & 22 & $-1,22$ & $-0,44$ & $-1,74$ & $-0,65$ & $-16,33^{*}$ & $-1,28$ & $-21,57^{*}$ & $-1,49$ \\
\hline 23. & 22 & $-2,46$ & $-0,86$ & $-2,15$ & $-0,75$ & $-18,49 *$ & $-1,61$ & $-29,15^{* * *}$ & $-3,07$ \\
\hline 24. & 22 & 1,14 & 0,40 & 1,32 & 0,44 & $-17,16^{*}$ & $-1,48$ & $-29,33 * * *$ & $-3,26$ \\
\hline 25. & 22 & $-2,24$ & $-0,73$ & $-3,75^{*}$ & $-1,41$ & $-20,92 * *$ & $-1,92$ & $-33,61 * * *$ & $-3,85$ \\
\hline \multicolumn{10}{|l|}{$\ldots$} \\
\hline 31. & 22 & 2,99 & 0,64 & 0,73 & 0,17 & $-29,71 * * *$ & $-2,46$ & $-40,89 * * *$ & $-4,02$ \\
\hline 32. & 22 & 0,37 & 0,24 & $-1,30$ & $-1,01$ & $-31,01 * * *$ & $-2,52$ & $-42,74 * * *$ & $-4,13$ \\
\hline 33. & 22 & $-0,94$ & $-0,78$ & $-0,53$ & $-0,47$ & $-31,54 * * *$ & $-2,51$ & $-43,15 * * *$ & $-4,08$ \\
\hline 34. & 22 & $-1,32$ & $-1,02$ & $-0,54$ & $-0,40$ & $-32,07 * * *$ & $-2,55$ & $-42,64 * * *$ & $-3,90$ \\
\hline 35. & 22 & 0,58 & 0,33 & 0,98 & 0,52 & $-31,10 * * *$ & $-2,45$ & $-39,25 * * *$ & $-3,02$ \\
\hline 36. & 22 & $3,16 * * *$ & 2,42 & $2,72^{*}$ & 1,54 & $-28,38 * *$ & $-2,26$ & $-36,04 * * *$ & $-2,45$ \\
\hline
\end{tabular}

Note: $\mathrm{n}$ represents the number of observations. Like Tarı's study (2012: 500), critical values for the t-test were taken as 1,282, 1,645 and 2,326 for the significance levels of $\% 10, \% 5$ and $\% 1$ respectively.

According to the findings in shown table 7, the averages returns of the industrial sector companies, which were offered to the public again in 2010 - 2014 period, were positive and statistically significant for the second month and fluctuated in the following months. In this case, it would be sensible to buy these stocks and sell them at the end of the second month. Average cumulative abnormal returns and average compound abnormal returns are generally negative, and these values are statistically significant since the 21th month. In this case, the hypothesis HO was accepted and it was decided that underperformance would be valid for stocks of industry sector. It has been seen that investors who hold these shares for 36 months have lost between $28 \%$ and $36 \%$ on average.

Table 8: Price Performance of Financial Firms' Shares for the first 36 Months

\begin{tabular}{|c|c|c|c|c|c|c|c|c|c|}
\hline Months & $\mathbf{n}$ & $\bar{R}$ & t- ist. & $\overline{A R}$ & t- ist. & $\overline{C A R}$ & t- ist. & $\overline{B H A R}$ & t- ist. \\
\hline 1. & 14 & $-6,75 * *$ & $-2,08$ & $-5,25 *$ & $-1,57$ & $-5,25^{*}$ & $-1,57$ & $-5,25 *$ & $-1,57$ \\
\hline 2. & 14 & $-6,85 * * *$ & $-2,67$ & $-5,23 * * *$ & $-2,62$ & $-10,49 * *$ & $-2,11$ & $-9,91 * *$ & $-2,16$ \\
\hline 3. & 14 & $-1,28$ & $-0,56$ & $-2,18$ & $-1,18$ & $-12,67 * *$ & $-2,17$ & $-11,54 * *$ & $-2,23$ \\
\hline 4. & 14 & 0,79 & 0,22 & 0,53 & 0,13 & $-12,14^{*}$ & $-1,59$ & $-11,15^{* *}$ & $-1,71$ \\
\hline 5. & 14 & $-2,74$ & $-0,80$ & $-1,11$ & $-0,31$ & $-13,25 *$ & $-1,35$ & $-11,29 *$ & $-1,29$ \\
\hline \multicolumn{10}{|l|}{... } \\
\hline 11. & 14 & $-1,92$ & $-0,64$ & $-1,32$ & $-0,51$ & $-21,67^{* *}$ & $-1,75$ & $-17,51^{* *}$ & $-1,78$ \\
\hline 12. & 14 & 1,89 & 0,90 & 0,51 & 0,23 & $-21,15^{* *}$ & $-1,67$ & $-17,75^{* *}$ & $-1,71$ \\
\hline 13. & 14 & $-1,92$ & $-0,97$ & $-3,17^{* *}$ & $-1,86$ & $-24,32^{* *}$ & $-1,94$ & $-19,57^{* *}$ & $-2,17$ \\
\hline 14. & 14 & 0,90 & 0,27 & 1,54 & 0,41 & $-22,78 * *$ & $-1,98$ & $-19,26 * * *$ & $-2,38$ \\
\hline 15. & 14 & $-1,91$ & $-0,97$ & $-2,45$ & $-1,14$ & $-25,22 * *$ & $-2,28$ & $-20,70 * * *$ & $-2,68$ \\
\hline \multicolumn{10}{|l|}{$\ldots$} \\
\hline 21. & 14 & $-1,07$ & $-0,47$ & $-1,98$ & $-0,89$ & $-39,90 * * *$ & $-2,68$ & $-31,23 * * *$ & $-2,94$ \\
\hline 22. & 14 & $-2,54$ & $-0,99$ & $-4,78^{*}$ & $-1,62$ & $-44,68 * * *$ & $-2,78$ & $-33,73 * * *$ & $-2,72$ \\
\hline
\end{tabular}




\begin{tabular}{l|lllllllll} 
23. & 14 & $5,70 *$ & 1,47 & 0,96 & 0,25 & $-43,72 * * *$ & $-2,65$ & $-34,99 * * *$ & $-2,51$ \\
24. & 14 & 2,88 & 0,94 & $3,09 *$ & 1,29 & $-40,63 * * *$ & $-2,40$ & $-32,53 * *$ & $-2,31$ \\
25. & 14 & $-2,10^{* *}$ & $-1,95$ & $-0,33$ & $-0,21$ & $-40,96 * * *$ & $-2,41$ & $-31,90^{* *}$ & $-2,29$ \\
.. & & & & & & & & & \\
31. & 14 & $-8,97 * * *$ & $-2,56$ & $-8,97 * * *$ & $-2,56$ & $-49,86 * * *$ & $-2,53$ & $-36,27 * * *$ & $-2,36$ \\
32. & 14 & $-6,41 * *$ & $-2,10$ & $-5,69 * *$ & $-1,85$ & $-55,55 * * *$ & $-2,89$ & $-40,98^{* * *}$ & $-2,82$ \\
33. & 14 & $-0,21$ & $-0,14$ & 0,76 & 0,56 & $-54,79 * * *$ & $-2,80$ & $-39,11^{* * *}$ & $-2,69$ \\
34. & 14 & $5,26 * *$ & 1,68 & $4,22 *$ & 1,43 & $-50,57 * * *$ & $-2,44$ & $-35,32 * *$ & $-2,26$ \\
35. & 14 & 1,99 & 1,20 & 1,01 & 0,48 & $-49,56 * *$ & $-2,29$ & $-34,91^{* *}$ & $-2,12$ \\
36. & 14 & $-1,47$ & $-0,36$ & $-3,16$ & $-0,80$ & $-52,72 * *$ & $-2,27$ & $-35,28^{* *}$ & $-1,95$ \\
\hline
\end{tabular}

Note: $\mathrm{n}$ represents the number of observations. Like Tarı's study (2012: 500), critical values for the t-test were taken as 1,282, 1,645 and 2,326 for the significance levels of $\% 10, \% 5$ and $\% 1$ respectively.

According to the findings shown in Table 8, the average returns of stocks in the financial sector, in 2010 - 2014 period, are generally negative and statistically significant. Especially cumulative abnormal returns and compound abnormal returns are negative for 36 months and statistically significant. In this case, the HO hypothesis was accepted and it was decided that the underperformance for the financial sector stocks in the period 2010-2014 was valid. It has been seen that investors who hold these shares for 36 months will suffer losses on an average of $35 \%$ to $53 \%$.

Table 9: Price Performance of Service Firms' Shares for the first 36 Months

\begin{tabular}{|c|c|c|c|c|c|c|c|c|c|}
\hline Months & n & $\bar{R}$ & t- ist. & $\overline{A R}$ & t- ist. & $\overline{C A R}$ & t- ist. & $\overline{B H A R}$ & t- ist. \\
\hline 1. & 6 & $-1,41$ & $-0,27$ & $-5,10$ & $-0,67$ & $-5,10$ & $-0,67$ & $-5,10$ & $-0,67$ \\
\hline 2. & 6 & $-4,66 * *$ & $-1,85$ & $-7,85^{* *}$ & $-1,82$ & $-12,94 * *$ & $-1,81$ & $-12,98 * *$ & $-1,84$ \\
\hline 3. & 6 & $-1,08$ & $-0,51$ & $-0,30$ & $-0,07$ & $-13,24^{* *}$ & $-1,84$ & $-12,83 * *$ & $-1,80$ \\
\hline 4. & 6 & 2,83 & 0,46 & 1,52 & 0,23 & $-11,73^{*}$ & $-1,30$ & $-12,17^{*}$ & $-1,45$ \\
\hline 5. & 6 & $-1,44$ & $-0,33$ & $-2,43$ & $-0,83$ & $-14,15^{*}$ & $-1,30$ & $-14,26^{*}$ & $-1,39$ \\
\hline \multicolumn{10}{|l|}{$\ldots$} \\
\hline 11. & 6 & $-7,10^{* *}$ & $-1,82$ & $-7,38 * * *$ & $-2,45$ & $-23,22$ & $-1,19$ & $-26,21^{*}$ & $-1,37$ \\
\hline 12. & 6 & $-2,92$ & $-0,95$ & $-4,14 * * *$ & $-5,23$ & $-27,36 *$ & $-1,44$ & $-28,83^{* *}$ & $-1,71$ \\
\hline 13. & 6 & 3,24 & 0,96 & 0,45 & 0,12 & $-26,92^{*}$ & $-1,38$ & $-27,35^{*}$ & $-1,51$ \\
\hline 14. & 6 & $-4,38 * * *$ & $-2,99$ & $-2,47$ & $-1,05$ & $-29,38^{*}$ & $-1,54$ & $-28,19 *$ & $-1,62$ \\
\hline 15. & 6 & $-2,61$ & $-1,26$ & $-5,68 * * *$ & $-3,43$ & $-35,07 * *$ & $-1,74$ & $-33,65^{* *}$ & $-1,87$ \\
\hline \multicolumn{10}{|l|}{$\ldots$} \\
\hline 21. & 6 & $-2,64 * *$ & $-1,74$ & $-2,85^{*}$ & $-1,40$ & $-26,23$ & $-1,22$ & $-21,84$ & $-1,19$ \\
\hline 22. & 6 & 2,26 & 0,92 & $-2,76$ & $-0,95$ & $-28,99 *$ & $-1,28$ & $-24,61^{*}$ & $-1,28$ \\
\hline 23. & 6 & $-0,10$ & $-0,06$ & 1,39 & 0,46 & $-27,61$ & $-1,11$ & $-21,60$ & $-0,99$ \\
\hline 24. & 6 & $-0,30$ & $-0,19$ & 0,63 & 0,20 & $-26,97$ & $-0,97$ & $-19,54$ & $-0,78$ \\
\hline 25. & 6 & $-2,10$ & $-0,35$ & $-3,68$ & $-0,75$ & $-30,65$ & $-1,09$ & $-24,73$ & $-0,98$ \\
\hline \multicolumn{10}{|l|}{$\ldots$} \\
\hline 31. & 6 & $-1,00$ & $-0,41$ & $-5,75 * *$ & $-2,20$ & $-54,69 * *$ & $-1,97$ & $-53,21 * *$ & $-2,32$ \\
\hline 32. & 6 & $-0,27$ & $-0,18$ & $-1,93$ & $-0,84$ & $-56,62 * *$ & $-2,01$ & $-54,91 * *$ & $-2,29$ \\
\hline 33. & 6 & $-3,85^{* *}$ & $-1,80$ & 0,54 & 0,22 & $-56,08 * *$ & $-2,05$ & $-52,54 * *$ & $-2,31$ \\
\hline 34. & 6 & $-1,72$ & $-0,37$ & $-0,58$ & $-0,12$ & $-56,65^{* *}$ & $-1,84$ & $-50,57 * *$ & $-1,83$ \\
\hline 35. & 6 & 12,20 & 1,03 & 11,00 & 0,96 & $-45,66$ & $-1,23$ & $-35,93$ & $-0,84$ \\
\hline 36. & 6 & 1,73 & 0,49 & $-0,81$ & $-0,39$ & $-46,47$ & $-1,20$ & $-31,26$ & $-0,64$ \\
\hline
\end{tabular}

Note: $\mathrm{n}$ represents the number of observations. Like Tarı's study (2012: 500), critical values for the t-test were taken as 1,282, 1,645 and 2,326 for the significance levels of $\% 10, \% 5$ and $\% 1$ respectively.

According to the findings in table 9, the average returns of the stocks in the services sector, which were offered in the period of 2010-2014, are generally negative and statistically significant, but the negative returns calculated at the 36th month cannot be fully confirmed in terms of statistically. In this case, it would be impossible to accept the HO hypothesis under the assumption that the investor holds the stock for a period of 36 months. It may be the rational investment decision to buy these shares at the date of issuance and sell them at the end of the 7th month may be a rational investment decision. However, it seems that an investor who holds these shares for 36 months will suffer an average loss of more than $50 \%$. 
Table 10: Price Performance of Construction Firms' Shares for the first 36 Months

\begin{tabular}{|c|c|c|c|c|c|c|c|c|c|}
\hline Months & $\mathrm{n}$ & $\bar{R}$ & t- ist. & $\overline{A R}$ & t- ist. & $\overline{C A R}$ & t- ist. & $\overline{B H A R}$ & t- ist. \\
\hline 1. & 3 & $-22,25^{* *}$ & $-1,90$ & $-22,14^{*}$ & $-1,62$ & $-22,14^{*}$ & $-1,62$ & $-22,14^{* *}$ & $-1,62$ \\
\hline 2. & 3 & $-8,85 * * *$ & $-5,45$ & $-8,13 * * *$ & $-2,98$ & $-30,28 * *$ & $-2,03$ & $-28,71^{*}$ & $-2,04$ \\
\hline 3. & 3 & $-5,15^{*}$ & $-1,35$ & $-3,96$ & $-1,01$ & $-34,24 * * *$ & $-3,11$ & $-31,71 * * *$ & $-2,86$ \\
\hline 4. & 3 & 3,08 & 0,89 & $-1,01$ & $-1,03$ & $-35,25 * * *$ & $-3,26$ & $-32,89 * * *$ & $-3,23$ \\
\hline 5. & 3 & 3,76 & 0,36 & 3,97 & 0,52 & $-31,27^{* *}$ & $-1,94$ & $-29,30 * *$ & $-2,18$ \\
\hline \multicolumn{10}{|l|}{... } \\
\hline 11. & 3 & $-4,88$ & $-0,52$ & $-3,76$ & $-0,99$ & $-46,13 * * *$ & $-2,70$ & $-40,22 * * *$ & $-3,03$ \\
\hline 12. & 3 & $-2,25^{*}$ & $-1,44$ & $-5,80 * * *$ & $-2,46$ & $-51,94 * * *$ & $-3,15$ & $-44,99 * * *$ & $-3,66$ \\
\hline 13. & 3 & $-3,84^{*}$ & $-1,38$ & $-4,31 * *$ & $-1,68$ & $-56,25 * * *$ & $-3,72$ & $-47,67 * * *$ & $-4,79$ \\
\hline 14. & 3 & $-4,67 * *$ & $-2,20$ & $-1,37$ & $-1,18$ & $-57,62 * * *$ & $-3,59$ & $-46,82 * * *$ & $-4,42$ \\
\hline 15. & 3 & $-6,24 * * *$ & $-3,06$ & $-1,58$ & $-0,65$ & $-59,20 * * *$ & $-3,26$ & $-44,80 * * *$ & $-4,14$ \\
\hline \multicolumn{10}{|l|}{$\ldots$} \\
\hline 21. & 3 & $-4,29$ & $-1,18$ & $-4,81$ & $-1,16$ & $-54,50 * * *$ & $-4,99$ & $-44,16 * * *$ & $-5,99$ \\
\hline 22. & 3 & $5,37 * *$ & 2,29 & $-2,40^{*}$ & $-1,41$ & $-56,90 * * *$ & $-5,61$ & $-49,01 * * *$ & $-6,32$ \\
\hline 23. & 3 & $9,70 * * *$ & 5,62 & $5,67 * * *$ & 4,47 & $-51,23 * * *$ & $-5,02$ & $-48,12 * * *$ & $-5,34$ \\
\hline 24. & 3 & $-5,36 * *$ & $-1,67$ & $-1,25$ & $-0,20$ & $-52,48 * * *$ & $-3,83$ & $-46,29 * * *$ & $-4,38$ \\
\hline 25. & 3 & $-4,59 * * *$ & $-5,78$ & $-3,78 * *$ & $-1,70$ & $-56,26 * * *$ & $-3,99$ & $-48,19 * * *$ & $-4,49$ \\
\hline$\cdots$ & & & & & & & & & \\
\hline 31. & 3 & 1,32 & 0,31 & 0,16 & 0,04 & $-62,33 * * *$ & $-5,27$ & $-56,94 * * *$ & $-5,12$ \\
\hline 32. & 3 & $3,16^{*}$ & 1,49 & $3,16^{*}$ & 1,49 & $-63,51 * * *$ & $-4,47$ & $-60,48 * * *$ & $-4,25$ \\
\hline 33. & 3 & $-4,01$ & $-0,81$ & $-1,96$ & $-0,94$ & $-65,48 * * *$ & $-4,86$ & $-59,14 * * *$ & $-5,20$ \\
\hline 34. & 3 & 0,44 & 0,12 & 0,32 & 0,14 & $-65,16 * * *$ & $-4,75$ & $-59,01 * * *$ & $-5,31$ \\
\hline 35. & 3 & 0,67 & 0,27 & 2,58 & 0,98 & $-62,59 * * *$ & $-5,33$ & $-56,95 * * *$ & $-5,26$ \\
\hline 36. & 3 & 0,34 & 0,12 & $-2,38$ & $-0,93$ & $-64,97 * * *$ & $-4,60$ & $-59,72 * * *$ & $-4,74$ \\
\hline
\end{tabular}

Note: $\mathrm{n}$ represents the number of observations. Like Tarı's study (2012: 500), critical values for the t-test were taken as 1,282, 1,645 and 2,326 for the significance levels of $\% 10, \% 5$ and $\% 1$ respectively.

According to the findings shown in Table 10, the average returns of the stocks in the construction sector are generally negative and statistically significant. Particularly cumulative abnormal returns and compound abnormal returns were negative for 36 months and statistically significant. In this case, the $\mathrm{HO}$ hypothesis was accepted and it was decided that the underperformance anomaly for construction sector stocks, which were re-offered in 2010-2014, was valid. Although it may seem logical decision to buy these stocks and selling them at the end of the 7th month, it seems that investors who hold these stocks since on the date of issue for 36 months have lost more than $60 \%$ on average.

Table 11: Price Performance of Energy Firms' Shares for the first 36 Months

\begin{tabular}{|c|c|c|c|c|c|c|c|c|c|}
\hline Months & $n$ & $\bar{R}$ & t- ist. & $\overline{A R}$ & t- ist. & $\overline{C A R}$ & t- ist. & $\overline{\overline{B H A R}}$ & t- ist. \\
\hline 1. & 5 & $-0,93$ & $-0,11$ & $-0,39$ & $-0,05$ & $-0,39$ & $-0,05$ & $-0,39$ & $-0,05$ \\
\hline 2. & 5 & $-0,94$ & $-0,22$ & $-4,69$ & $-0,92$ & $-5,07$ & $-0,92$ & $-5,68$ & $-1,10$ \\
\hline 3. & 5 & $2,52^{*}$ & 1,47 & 1,10 & 0,29 & $-3,97$ & $-0,54$ & $-4,56$ & $-0,65$ \\
\hline 4. & 5 & $-2,71$ & $-0,79$ & $-2,25$ & $-0,85$ & $-6,22$ & $-0,99$ & $-7,62$ & $-1,24$ \\
\hline 5. & 5 & $-1,73$ & $-0,50$ & $-4,90 *$ & $-1,60$ & $-11,12 * *$ & $-1,89$ & $-13,08 * *$ & $-2,16$ \\
\hline$\ldots$ & & & & & & & & & \\
\hline 11. & 5 & 1,11 & 0,42 & 1,15 & 0,48 & $-12,18$ & $-0,71$ & $-11,78$ & $-0,75$ \\
\hline 12. & 5 & 5,98 & 1,16 & $-1,02$ & $-0,28$ & $-13,21$ & $-0,90$ & $-15,00$ & $-0,99$ \\
\hline 13. & 5 & $-7,43 * *$ & $-1,73$ & $-2,79$ & $-0,79$ & $-15,99 *$ & $-1,33$ & $-17,63^{*}$ & $-1,60$ \\
\hline 14. & 5 & $-4,88 * *$ & $-1,90$ & $-2,56$ & $-0,78$ & $-18,55^{*}$ & $-1,49$ & $-18,79 * *$ & $-1,77$ \\
\hline 15. & 5 & $-4,86^{*}$ & $-1,44$ & $-2,03$ & $-0,91$ & $-20,57^{* *}$ & $-1,66$ & $-20,02 * *$ & $-2,10$ \\
\hline$\ldots$ & & & & & & & & & \\
\hline 21. & 5 & $-2,91 * *$ & $-1,85$ & $-3,56 * *$ & $-1,80$ & $-44,31 * * *$ & $-3,30$ & $-39,09 * * *$ & $-4,15$ \\
\hline 22. & 5 & $4,58 * * *$ & 1,01 & $-0,76$ & $-0,25$ & $-45,07 * * *$ & $-3,35$ & $-42,16 * * *$ & $-4,17$ \\
\hline 23. & 5 & $5,58 * *$ & 1,72 & $-1,19$ & $-0,40$ & $-46,26 * * *$ & $-3,24$ & $-45,56 * * *$ & $-4,21$ \\
\hline 24. & 5 & $-1,17$ & $-0,55$ & $-0,99$ & $-0,47$ & $-47,25 * * *$ & $-3,07$ & $-45,16 * * *$ & $-4,09$ \\
\hline 25. & 5 & $-0,83$ & $-0,26$ & $-3,59$ & $-1,06$ & $-50,84 * * *$ & $-2,96$ & $-48,34 * * *$ & $-4,15$ \\
\hline
\end{tabular}




\begin{tabular}{l|lllllllll}
$\ldots$ & & & & & & & & & \\
31. & 5 & $-4,02$ & $-1,07$ & $-5,93^{*}$ & $-1,36$ & $-31,96$ & $-1,13$ & $-26,78$ & $-1,12$ \\
32. & 5 & $-1,18$ & $-0,46$ & $-4,92^{*}$ & $-1,61$ & $-36,88^{*}$ & $-1,39$ & $-34,25^{*}$ & $-1,54$ \\
33. & 5 & 6,26 & 0,91 & 7,45 & 1,11 & $-29,43^{*}$ & $-1,32$ & $-32,24^{*}$ & $-1,54$ \\
34. & 5 & $-3,84^{*}$ & $-1,43$ & $-1,04$ & $-0,41$ & $-30,48^{*}$ & $-1,42$ & $-33,19^{* *}$ & $-1,76$ \\
35. & 5 & $-0,06$ & $-0,03$ & 0,52 & 0,30 & $-29,95^{*}$ & $-1,36$ & $-32,47^{*}$ & $-1,65$ \\
36. & 5 & $-0,98$ & $-0,33$ & 1,45 & 0,58 & $-28,50^{*}$ & $-1,28$ & $-30,72^{*}$ & $-1,52$ \\
\hline
\end{tabular}

Note: $\mathrm{n}$ represents the number of observations. Like Tarı's study (2012: 500), critical values for the t-test were taken as 1,282, 1,645 and 2,326 for the significance levels of $\% 10, \% 5$ and $\% 1$ respectively.

According to the findings shown in Table 11., the average returns of stocks in the energy sector during the 2010 - 2014 period, are generally negative and statistically significant. In this case, the HO hypothesis was accepted and it was decided that underperformance for energy sector stocks, which were re-offered in 2010-2014, is valid. It seems a rational decision to buy these stocks and selling them at the end of the third month. However, it seems that investors who hold these stocks for 36 months on average during lost more than $30 \%$ on average.

\subsection{Peer Group Analysis}

In this part of the study, stocks performance of the firms that made offering to the public in the periods following initial public offerings for the period of 2010-2015, with their peer firms that match sample firms by asset size and M/B ratio will be compared. Thus, it will be tried to reveal the effects of the making seasoned equity offerings on the returns of the stocks. For this purpose, while the peer group of the companies included in the sample; firms that took place in the same sector and with a similar asset size and PD / DD multiply were selected.

Here, the average raw returns, the average abnormal returns, the average cumulative abnormal returns, and the average compound abnormal returns are calculated for the first 36 months after the public offering. For this purpose, the closing prices of the stocks and BIST National 100 index at the end of the month were used.

The hypotheses used at this stage of the analysis are;

$H_{0}: \bar{R} ; \overline{A R} ; \overline{C A R} ; \overline{B H A R} \leq 0$ Underperformance anomaly is valid according to average returns.

$H_{1}: \bar{R} ; \overline{A R} ; \overline{C A R} ; \overline{B H A R}>0$ Underperformance anomaly is not valid according to average returns.

In order to test these hypotheses, the average t-statistics are calculated by using standard deviations and the findings are presented in Table 12.

Table 12: Peer Group Analysis Results

\begin{tabular}{|c|c|c|c|c|c|c|c|c|c|}
\hline Months & $\mathbf{n}$ & $\bar{R}$ & t- ist. & $\overline{A R}$ & t- ist. & $\overline{C A R}$ & t- ist. & $\overline{B H A R}$ & t- ist. \\
\hline 1. & 20 & $-6,09 * *$ & $-1,72$ & $-7,16 * *$ & $-1,92$ & $-7,16 * *$ & $-1,96$ & $-7,16 * *$ & $-1,92$ \\
\hline 2. & 20 & $-1,33$ & $-0,80$ & $-1,15$ & $-0,55$ & $-8,42 * *$ & $-1,83$ & $-7,77^{* *}$ & $-1,72$ \\
\hline 3. & 20 & $-1,41$ & $-0,77$ & $-0,45$ & $-0,33$ & $-8,87 * *$ & $-1,98$ & $-8,22 * *$ & $-1,88$ \\
\hline 4. & 20 & $3,88^{*}$ & 1,37 & $3,66^{*}$ & 1,39 & $-5,21$ & $-0,95$ & $-4,49$ & $-0,82$ \\
\hline 5. & 20 & $2,91^{*}$ & 1,32 & 1,15 & 0,64 & $-4,06$ & $-0,70$ & $-3,35$ & $-0,55$ \\
\hline \multicolumn{10}{|l|}{$\ldots$} \\
\hline 11. & 20 & 0,43 & 0,15 & $-1,36$ & $-0,61$ & $-2,11$ & $-0,32$ & $-2,90$ & $-0,41$ \\
\hline 12. & 20 & 0,89 & 0,66 & $-0,30$ & $-0,19$ & $-2,41$ & $-0,33$ & $-2,15$ & $-0,26$ \\
\hline 13. & 20 & 0,42 & 0,23 & $-0,70$ & $-0,39$ & $-3,11$ & $-0,38$ & $-1,43$ & $-0,15$ \\
\hline 14. & 20 & $-2,11 * *$ & $-1,70$ & $-1,76^{*}$ & $-1,30$ & $-4,86$ & $-0,56$ & $-2,59$ & $-0,27$ \\
\hline 15. & 20 & $-0,97$ & $-0,70$ & $-0,39$ & $-0,29$ & $-5,25$ & $-0,55$ & $-2,26$ & $-0,21$ \\
\hline \multicolumn{10}{|l|}{$\ldots$} \\
\hline 21. & 20 & 0,76 & 0,78 & $-0,79$ & $-0,76$ & $-0,29$ & $-0,03$ & 2,85 & 0,24 \\
\hline 22. & 20 & 1,05 & 0,72 & 0,71 & 0,51 & 0,42 & 0,04 & 4,39 & 0,34 \\
\hline 23. & 20 & 2,53 & 1,26 & 1,76 & 0,97 & 2,18 & 0,23 & 7,02 & 0,49 \\
\hline 24. & 20 & $2,52^{*}$ & 1,43 & $3,32 * *$ & 1,85 & 5,50 & 0,54 & 11,34 & 0,77 \\
\hline 25. & 20 & 0,22 & 0,19 & $-0,10$ & $-0,10$ & 5,40 & 0,51 & 11,70 & 0,79 \\
\hline $\begin{array}{l}\cdots \\
31 .\end{array}$ & 20 & $2,80 * * *$ & 2,58 & 0,96 & 0,75 & 0,10 & 0,01 & 6,49 & 0,43 \\
\hline 31. & 20 & 2,00 & 2,30 & 0,90 & 0,15 & 0,10 & 0,01 & 6,49 & 0,43 \\
\hline
\end{tabular}




\begin{tabular}{l|lllllllll} 
32. & 20 & 1,75 & 1,09 & 0,67 & 0,44 & 0,78 & 0,07 & 7,32 & 0,47 \\
33. & 20 & $-0,55$ & $-0,47$ & 0,95 & 0,83 & 1,72 & 0,15 & 8,97 & 0,59 \\
34. & 20 & 2,09 & 1,05 & 1,50 & 0,78 & 3,22 & 0,26 & 11,48 & 0,72 \\
35. & 20 & 2,05 & 1,16 & 0,94 & 0,54 & 4,16 & 0,32 & 12,82 & 0,77 \\
36. & 20 & 1,84 & 1,15 & 0,54 & 0,37 & 4,70 & 0,35 & 14,68 & 0,81 \\
\hline
\end{tabular}

Note: $\mathrm{n}$ represents the number of observations. Like Tarı's study (2012: 500), critical values for the t-test were taken as 1,282, 1,645 and 2,326 for the significance levels of $\% 10, \% 5$ and $\% 1$ respectively.

According to the results shown in Table 12, the average raw returns and average abnormal returns were found to be negative and statistically significant in the first month. Average cumulative abnormal returns and average compound abnormal returns were found to be negative and statistically significant in the first three months. In this case, $\mathrm{H}_{-} \mathrm{O}$ hypotheses were accepted at the level of $5 \%$ significance level for the first 3 months and it was determined that underperformance anomaly is valid for sample firms compared to the peer group. When the average raw returns are examined; 4th, 5th, 6th, 16th, 17th, 24th, and 31st months returns were found to be positively and statistically significant. Average abnormal returns were positive and statistically significant at the 4 th, 8 th, 9 th and 24 th months.

It is possible to reject the $\mathrm{H}_{-} 0$ hypotheses related to abnormal returns and raw returns observed in these months, and it can be said that the high price-performance case was experienced in those months. However, these results are not supported by cumulative abnormal returns compound abnormal returns.

Comparative results of long-term price performance analysis for the companies that perform seos in the main sample and those that do not soes in the peer group are presented in Table 13.

Table 13: The Effect of Seasoned Equity Offerings Conducted During The 2010-2014 Period on the Long-Term Share Price Performance

\begin{tabular}{|c|c|c|c|c|c|c|c|c|}
\hline \multirow{2}{*}{ Months } & \multicolumn{4}{|c|}{ Main Group } & \multicolumn{4}{|c|}{ Peer Group } \\
\hline & $\bar{R}$ & $\overline{A R}$ & $\overline{C A R}$ & $\overline{B H A R}$ & $\overline{\boldsymbol{R}}$ & $\overline{A R}$ & $\overline{C A R}$ & $\overline{B H A R}$ \\
\hline 1. & $-2,10$ & $-2,95$ & $-2,95$ & $-2,95$ & $-6,09 * *$ & $-7,16 * *$ & $-7,16 * *$ & $-7,16 * *$ \\
\hline 2. & 0,23 & $-0,14$ & $-3,09$ & $-3,60$ & $-1,33$ & $-1,15$ & $-8,42 * *$ & $-7,77 * *$ \\
\hline 3. & $-0,11$ & $-0,77$ & $-3,86$ & $-4,22$ & $-1,41$ & $-0,45$ & $-8,87 * *$ & $-8,22 * *$ \\
\hline 4. & 1,03 & $-0,18$ & $-4,05$ & $-5,51$ & $3,88^{*}$ & $3,66^{*}$ & $-5,21$ & $-4,49$ \\
\hline 5. & $-2,09$ & $-3,09 * *$ & $-7,13^{*}$ & $-9,20 * *$ & $2,91^{*}$ & 1,15 & $-4,06$ & $-3,35$ \\
\hline \multicolumn{9}{|l|}{$\ldots$} \\
\hline 11. & $-0,86$ & $-1,54$ & $-12,32^{* *}$ & $-8,91$ & 0,43 & $-1,36$ & $-2,11$ & $-2,90$ \\
\hline 12. & $-0,82$ & $-2,41 * * *$ & $-14,72 * *$ & $-12,19 *$ & 0,89 & $-0,30$ & $-2,41$ & $-2,15$ \\
\hline 13. & $-1,52^{*}$ & $-2,22 * * *$ & $-16,95 * * *$ & $-14,64 * *$ & 0,42 & $-0,70$ & $-3,11$ & $-1,43$ \\
\hline 14. & $-2,85 * *$ & $-1,94$ & $-18,89 * * *$ & $-16,37^{* *}$ & $-2,11^{* *}$ & $-1,76^{*}$ & $-4,86$ & $-2,59$ \\
\hline 15. & $-3,63 * * *$ & $-2,67 * * *$ & $-21,56 * * *$ & $-18,03 * *$ & $-0,97$ & $-0,39$ & $-5,25$ & $-2,26$ \\
\hline \multicolumn{9}{|l|}{$\ldots$} \\
\hline 21. & $-0,96$ & $-1,81^{* *}$ & $-28,44 * * *$ & $-24,79 * * *$ & 0,76 & $-0,79$ & $-0,29$ & 2,85 \\
\hline 22. & $-0,19$ & $-2,66^{* *}$ & $-31,10^{* * *}$ & $-29,05 * * *$ & 1,05 & 0,71 & 0,42 & 4,39 \\
\hline 23. & 1,64 & $-0,29$ & $-31,39 * * *$ & $-32,66 * * *$ & 2,53 & 1,76 & 2,18 & 7,02 \\
\hline 24. & 0,83 & 1,35 & $-30,04 * * *$ & $-31,65 * * *$ & $2,52^{*}$ & $3,32 * *$ & 5,50 & 11,34 \\
\hline 25. & $-2,18^{*}$ & $-2,77^{* *}$ & $-32,81 * * *$ & $-34,41 * * *$ & 0,22 & $-0,10$ & 5,40 & 11,70 \\
\hline \multicolumn{9}{|l|}{... } \\
\hline 31. & $-1,64$ & $-3,10^{*}$ & $-40,53 * * *$ & $-40,63 * * *$ & $2,80 * * *$ & 0,96 & 0,10 & 6,49 \\
\hline 32. & $-1,59 *$ & $-2,96 * * *$ & $-43,49 * * *$ & $-43,93 * * *$ & 1,75 & 0,67 & 0,78 & 7,32 \\
\hline 33. & $-0,55$ & 0,67 & $-42,82 * * *$ & $-43,02 * * *$ & $-0,55$ & 0,95 & 1,72 & 8,97 \\
\hline 34. & 0,33 & 0,79 & $-42,03 * * *$ & $-41,58 * * *$ & 2,09 & 1,50 & 3,22 & 11,48 \\
\hline 35. & $2,31 *$ & $2,24 *$ & $-39,79 * * *$ & $-38,02 * * *$ & 2,05 & 0,94 & 4,16 & 12,82 \\
\hline 36. & 1,11 & 0,22 & $-39,57 * * *$ & $-36,14 * * *$ & 1,84 & 0,54 & 4,70 & 14,68 \\
\hline
\end{tabular}

Note: $\mathrm{n}$ represents the number of observations. Like Tarı's study (2012: 500), critical values for the t-test were taken as 1,282, 1,645 and 2,326 for the significance levels of $\% 10, \% 5$ and $\% 1$ respectively.

When the results in Table 13 are compared, it is seen that there are significant differences between the long-term price performances of the two groups' stocks. In the first quarter, the stocks of the firms in both groups were also damaged, while the results of the main group were statistically insignificant. In the fifth, eighth and ninth months, the peer group provide profits to 
its investors while investors in the stocks of the main group of companies had a loss. The average cumulative abnormal returns and the average compound returns in the main group were negative and statistically significant, while the returns of the peer group were wavy and statistically insignificant. In general, it is observed that the peer group firms' long-term stock performance is significantly higher than the stock performance of the firms included in the sample. This supports long-term underperformance anomalies during seos. For all these reasons; it has been decided that performing seos is an important determinant of the long-term price performance of equities.

\subsection{Determinants of the long-term price performance of equities}

At this stage of the analysis, regression analyzes were performed using AR and CAR values calculated in previous analyzes as dependent variables and other relevant data as independent variables.

Variables and hypotheses used in these analyzes are shown in Table 14.

Table 14: List of Independent Variables

\begin{tabular}{|c|c|}
\hline Offering Price (LnOP) & Prices the stocks are offered to the public \\
\hline Public Offering Frequency (POF) & Frequency of the offering during the period. \\
\hline Volatility (V) & Standard deviations of stocks \\
\hline Asset Size (LnASSET) & Assets of the firms based on IFRS. \\
\hline Offering Method (om) & Public \& Private placement. \\
\hline Volume (LnVol) & $\begin{array}{l}\text { Total revenues come from the trading of the shares } \\
\text { during }\end{array}$ \\
\hline Difference (DF) & $\begin{array}{l}\text { Difference between obtained income and target } \\
\text { income }\end{array}$ \\
\hline Leverage Change (LC) & Total Liabilities / Total Assets \\
\hline Capital Increase Rate (CIR) & $\begin{array}{l}\text { The increase as \% change compared to previous } \\
\text { capital. }\end{array}$ \\
\hline Market to Book Value Ratio (M/B) & Market Value / Book Value \\
\hline
\end{tabular}

In order to prevent the problem of varying variance as a result of the analysis, the offer price, asset size and volume variables were included in the analyzes by taking the natural logarithm. AR and CAR values were used as dependent variables in this analysis. Volatility change was used as the standard deviation ( $\left.V_{-} A R\right)$ of the AR and standard deviation (V_CAR). The econometric models that are used in the analysis through AR and CAR dependent variable are shown below. The models were set up as one explanatory variable to test the effects of all variables separately.

$$
\begin{aligned}
& \text { Model (1): } A R_{i t} \& C A R_{i t}=\beta_{0}+\beta_{1} H A F_{i t}+e_{i t} \\
& \text { Model (2): } A R_{i t} \& C A R_{i t}=\beta_{0}+\beta_{1} H A A_{i t}+e_{i t} \\
& \text { Model (3): } A R_{i t} \& C A R_{i t}=\beta_{0}+\beta_{1} L n A S S E T_{i t}+e_{i t} \\
& \text { Model (4): } A R_{i t} \& C A R_{i t}=\beta_{0}+\beta_{1} K_{H A M 1_{i t}}+e_{i t} \\
& \text { Model (5): } A R_{i t} \& C A R_{i t}=\beta_{0}+\beta_{1} K_{H A M 2_{i t}}+e_{i t} \\
& \text { Model (6): } A R_{i t} \& C A R_{i t}=\beta_{0}+\beta_{1} L n I H_{i t}+e_{i t} \\
& \text { Model (7): } A R_{i t} \& C A R_{i t}=\beta_{0}+\beta_{1} F A R K_{i t}+e_{i t} \\
& \text { Model (8): } A R_{i t} \& C A R_{i t}=\beta_{0}+\beta_{1} K O D_{i t}+e_{i t} \\
& \text { Model (9): } A R_{i t} \& C A R_{i t}=\beta_{0}+\beta_{1} S A O_{i t}+e_{i t} \\
& \text { Model (10): } A R_{i t} \& C A R_{i t}=\beta_{0}+\beta_{1} P D / D D_{i t}+e_{i t} \\
& \text { Model (11): } A R_{i t} \& C A R_{i t}=\beta_{0}+\beta_{1} V_{-} A R_{i t}+e_{i t}
\end{aligned}
$$

Since the time dimension is enough models are estimated by panel data analysis method.

The steps followed in the analysis are as follows:

* Panel unit root test used to determine stationarity level of the series, 
* In order to see the existence of the interaction between the series in each model, panel causality test was conducted,

* Panel cointegration test was performed to determine whether the series was moving together in the long run,

* Finally according to previous analysis results panel regression analyzes were performed.

In this context, firstly, the panel unit root test was performed and it was tested whether the series were stationary. The stability of the series was examined by Im, Pesaran and Shin (2003) (IPS). These tests are based on the following model:

$$
\Delta Y_{i, t}=\delta_{i} Y_{i, t-1}+\sum_{j=1}^{p_{i}} \beta_{i j} \Delta Y_{i, t-j}+X_{i, t}^{\prime} \theta+\varepsilon_{i, t}
$$

$p_{i}$; represents the optimum lag length,

$X_{i, t}^{\prime}$; refers to external variables that contain any fixed effect or individual trend component.

In the panel unit root tests, it is tried to determine how the value of the series at the time $t$ is affected by the value at the time $t$ 1. In IPS (2003) test, it is accepted that $\delta_{-} i$, which is a unit root parameter, may be different between the series.

IPS panel unit root test was performed in the study and the results obtained are presented in Table 15.

Table 15: Panel Unit Root Test Results

\begin{tabular}{|c|c|c|c|c|}
\hline \multirow{2}{*}{$\begin{array}{l}\text { Independent } \\
\text { Varible }\end{array}$} & \multicolumn{2}{|c|}{ Original Level Values } & \multicolumn{2}{|c|}{ First Differences of the Series } \\
\hline & Test Statistic & Prob.Value & Test Statistic & Prob.Value \\
\hline AR & -1.12 & 0.13 & $-45.27 * * *$ & 0.00 \\
\hline CAR & 0.25 & 0.60 & $-27.99 * * *$ & 0.00 \\
\hline LnOP & \multirow{5}{*}{\multicolumn{4}{|c|}{$\begin{array}{l}\text { The unit root test cannot be performed because the series has the same values throughout the analysis } \\
\text { period, therefore, it is accepted that the series is stationary because it is composed of fixed values. } \\
\text { The unit root test cannot be performed because the series has the same values throughout the analysis } \\
\text { period, therefore, it is accepted that the series is stationary because it is composed of fixed values. } \\
\text { The unit root test cannot be performed because the series has the same values throughout the analysis } \\
\text { period, therefore, it is accepted that the series is stationary because it is composed of fixed values. } \\
\text { The unit root test cannot be performed because the series has the same values throughout the analysis } \\
\text { period, therefore, it is accepted that the series is stationary because it is composed of fixed values. } \\
\text { The unit root test cannot be performed because the series has the same values throughout the analysis } \\
\text { period, therefore, it is accepted that the series is stationary because it is composed of fixed values. }\end{array}$}} \\
\hline POF & & & & \\
\hline LnASSET & & & & \\
\hline $\begin{array}{l}\text { Public } \\
\text { Placement }\end{array}$ & & & & \\
\hline $\begin{array}{l}\text { Private } \\
\text { Placement }\end{array}$ & & & & \\
\hline LnVol & $-1,27$ & 0.10 & $-17.95 * * *$ & 0.00 \\
\hline DF & \multirow{4}{*}{\multicolumn{4}{|c|}{$\begin{array}{l}\text { The unit root test cannot be performed because the series has the same values throughout the analysis } \\
\text { period, therefore, it is accepted that the series is stationary because it is composed of fixed values. } \\
\text { The unit root test cannot be performed because the series has the same values throughout the analysis } \\
\text { period, therefore, it is accepted that the series is stationary because it is composed of fixed values. } \\
\text { The unit root test cannot be performed because the series has the same values throughout the analysis } \\
\text { period, therefore, it is accepted that the series is stationary because it is composed of fixed values. } \\
\text { The unit root test cannot be performed because the series has the same values throughout the analysis } \\
\text { period, therefore, it is accepted that the series is stationary because it is composed of fixed values. }\end{array}$}} \\
\hline LC & & & & \\
\hline CIR & & & & \\
\hline$M / B$ & & & & \\
\hline V_AR & 1.40 & 0.92 & $-22.59 * * *$ & 0.00 \\
\hline V_CAR & 2.62 & 0.99 & $-30.09 * * *$ & 0.00 \\
\hline
\end{tabular}

Note: $*, * *$ and $* * *$ indicate stationary at the level of significance of $10 \%, 5 \%$ and $1 \%$, respectively. Fixed and trendy model was used for the test for the original level of the series, and the fixed model was used for the test for the first differences. The ideal lag length is determined according to the Schwarz information criterion.

According to the findings in Table 15, all series are not stationary in their original levels and become stationary at the \%1 significant level when the first differences are taken. Therefore, it is available to infer that even in a 36-months period, the series contained significant fluctuations. In this case, the regression analyzes that performed with the original level values of these 
series may contain the spurious regression problem. For this reason, it is necessary to perform the cointegration test before performing to the regression analysis. Before performing the cointegration test, a panel causality test was conducted to measure the interaction between the series that were found to be non-stationary in the unit root tests. In this study, the existence of causality relations among the series was examined by Granger (1969) panel causality test and findings are shown in the 16 Table.

Table 16: Panel Causality Test Results

\begin{tabular}{|c|c|c|c|}
\hline Model & $t_{0}$ Hypothesis & i-statistic & Prob.Value \\
\hline Model 6 & $\operatorname{LnIH} \nrightarrow A R$ & $.29 * *$ & 1.03 \\
\hline Model 11 & $V \_A R \nrightarrow A R$ & .69 & 1.18 \\
\hline Model 6 & $L n I H \nrightarrow C A R$ & $.32 * *$ & 1.01 \\
\hline nodel 11 & $V_{-} C A R \nrightarrow C A R$ & .12 & 1.12 \\
\hline
\end{tabular}

Note: * and $* *$ indicate causality relation from the first variable to second one at the level of significance of $10 \%$ and $5 \%$, respectively. The ideal lag length is determined according to the Schwarz information criterion.

According to the results represented in Table 16, we observed causality relationship between transaction volume and abnormal returns at $5 \%$ significance level while we couldn't find causality relation between volatility and abnormal returns. Panel cointegration test was applied to determine whether the non-stationary series move together in the long run. In this study, the existence of cointegration among the series was examined by the Perdroni (2004) test.

Table 17: Panel Cointegration Test Results for Model (6) (Dependent Variable AR)

\begin{tabular}{lllll}
\hline \hline & Test & Prob. & $\begin{array}{l}\text { Weighted Test } \\
\text { Statistic }\end{array}$ & $\begin{array}{l}\text { Prob. } \\
\text { Value }\end{array}$ \\
\hline Panel $\boldsymbol{v}$ & 0.47 & 0.31 & -1.25 & 0.89 \\
Panel $\boldsymbol{\rho}$ & $-9.92^{* * *}$ & 0.00 & $-11.76 * * *$ & 0.00 \\
Panel $\boldsymbol{t}$ & $-9.26^{* * *}$ & 0.00 & $-11.04 * * *$ & 0.00 \\
Panel $\boldsymbol{A D F}$ & $-9.01 * * *$ & 0.00 & $-10.88^{* * *}$ & 0.00 \\
Group $\boldsymbol{\rho}$ & $-7.14^{* * *}$ & 0.00 & - & - \\
Group $\boldsymbol{t}$ & $-9.05^{* * *}$ & 0.00 & - & - \\
Group $\boldsymbol{A D F}$ & $-8.47^{* * *}$ & 0.00 & - & - \\
\hline
\end{tabular}

Note: ${ }^{* * *}$ and $* *$ indicate cointegration relationship at the level of significance of $1 \%$ and $5 \%$, respectively.

According to the results shown in table 17, there is a cointegration relationship between volume and abnormal returns at the level of $1 \%$ significance. In other words, these series are moving together in the long run, and the model predictions to be made with the original level values of these series will not contain the spurious regression problem.

Table 18: Panel Cointegration Test Results for Model (11) (Dependent Variable AR)

\begin{tabular}{|c|c|c|c|c|}
\hline & $\begin{array}{l}\text { Test } \\
\text { Statistic }\end{array}$ & $\begin{array}{l}\text { Prob. } \\
\text { Valu }\end{array}$ & $\begin{array}{l}\text { Weighted } \\
\text { Test }\end{array}$ & $\begin{array}{l}\text { Prob. } \\
\text { Valu }\end{array}$ \\
\hline Panel $v$ & $4.36 * * *$ & 0.00 & -1.13 & 0.87 \\
\hline Panel $\rho$ & $28.75^{* * *}$ & 0.00 & $-25.34 * * *$ & 0.00 \\
\hline Panel $t$ & $\begin{array}{l}- \\
26.60 * * *\end{array}$ & 0.00 & $-24.16 * * *$ & 0.00 \\
\hline Panel ADF & $26.58 * * *$ & 0.00 & $-24.69 * * *$ & 0.00 \\
\hline Group $\rho$ & $22.45 * * *$ & 0.97 & - & - \\
\hline Group $t$ & $31.92 * * *$ & 0.00 & - & - \\
\hline Group $A D F$ & $29.86 * * *$ & 0.00 & - & - \\
\hline
\end{tabular}

Note: $* * *$ indicate cointegration relationship at the level of significance of $1 \%$. 
According to the results shown in table 18 , there is a cointegration relationship between volatility and abnormal returns at the level of $1 \%$ significance. In other words, these series are moving together in the long run, and the model predictions to be made with the original level values of these series will not contain the spurious regression problem.

Table 19: Panel Cointegration Test Results for Model (6) (Dependent Variable CAR)

\begin{tabular}{|c|c|c|c|c|}
\hline & $\begin{array}{l}\text { Test } \\
\text { Statistic }\end{array}$ & $\begin{array}{l}\text { Prob. } \\
\text { Valu }\end{array}$ & $\begin{array}{l}\text { Weighted } \\
\text { Test }\end{array}$ & $\begin{array}{l}\text { Prob. } \\
\text { Valu }\end{array}$ \\
\hline Panel $v$ & 0.003 & 0.49 & -1.35 & 0.91 \\
\hline Panel $\rho$ & $\begin{array}{l}- \\
2.49 * * *\end{array}$ & 0.00 & $-2.19 * *$ & 0.01 \\
\hline Panel $t$ & $3.45 * * *$ & 0.00 & $-3.29 * * *$ & 0.00 \\
\hline Panel ADF & 3.66*** & 0.00 & $-3.83 * * *$ & 0.00 \\
\hline Group $\rho$ & 0.25 & 0.60 & - & - \\
\hline Group $t$ & $1.89 * * *$ & 0.02 & - & - \\
\hline Group $A D F$ & $2.47 * * *$ & 0.00 & - & - \\
\hline
\end{tabular}

Note: ${ }^{* *}$ and $* *$ indicate cointegration relationship at the level of significance of $1 \%$ and $5 \%$, respectively.

According to the results shown in table 19 , there is a cointegration relationship between volume and cumulative abnormal returns at the level of $1 \%$ significance. In other words, these series are moving together in the long run, and the model predictions to be made with the original level values of these series will not contain the spurious regression problem.

Table 20: Panel Cointegration Test Results for Model (11) (Dependent Variable CAR)

\begin{tabular}{l|llll}
\hline \hline & $\begin{array}{l}\text { Test } \\
\text { Statistic }\end{array}$ & $\begin{array}{l}\text { Prob. } \\
\text { Valu }\end{array}$ & $\begin{array}{l}\text { Weighted } \\
\text { Test }\end{array}$ & $\begin{array}{l}\text { Prob. } \\
\text { Valu }\end{array}$ \\
\hline Panel $\boldsymbol{v}$ & $2.73^{* * *}$ & 0.00 & 2.59 & 0.00 \\
Panel $\rho$ & - & & & \\
Panel $\boldsymbol{t}$ & $2.49^{* * *}$ & 0.76 & $-3.00^{* * *}$ & 0.00 \\
Panel $\boldsymbol{A D F}$ & $\begin{array}{l}2.98^{* * *} \\
-\end{array}$ & 0.00 & $-3.25^{* * *}$ & 0.00 \\
Group $\boldsymbol{\rho}$ & $3.39^{* * *}$ & 0.00 & $-3.83^{* * *}$ & 0.00 \\
Group $\boldsymbol{t}$ & -0.30 & 0.37 & - & - \\
Group $\boldsymbol{A D F}$ & $-1.70^{* *}$ & 0.04 & - & - \\
\hline
\end{tabular}

Note: ${ }^{* * *}$ and $* *$ indicate cointegration relationship at the level of significance of $1 \%$ and $5 \%$, respectively.

According to the results shown in table 20 , there is a cointegration relationship between volatility and cumulative abnormal returns at the level of $1 \%$ significance. In other words, these series are moving together in the long run, and the model predictions to be made with the original level values of these series will not contain the spurious regression problem.

At this stage of the analysis, regression analysis will be performed. The regression analyzes were performed using the Panel Dynamic Ordinary Least Squares (PDOLS) method, which considers cointegration relations between the series and avoids the problems by using pre and lag values in the estimating model. The results of modeling the dependent variable AR and CAR are presented in Table 21 and Table 22 respectively. 
Table 21: First 36-Months Price Performance Analysis Results (Dependent Variable - AR)

\begin{tabular}{|c|c|c|c|c|c|c|c|c|c|c|c|}
\hline $\begin{array}{l}\text { Independent } \\
\text { Varible }\end{array}$ & Model 1 & Model 2 & Model 3 & Model 4 & Model 5 & 5Model & 6Model 7 & 7Model & BModel 9 & Model 10 & Model 11 \\
\hline LnOP & $\begin{array}{l}-0.84 * * * \\
{[-3.30]}\end{array}$ & - & - & - & - & - & - & - & - & - & - \\
\hline POF & - & $\begin{array}{l}-1.08 * * * \\
{[-3.89]}\end{array}$ & - & - & - & - & - & - & - & - & - \\
\hline LnASSET & - & - & $\begin{array}{l}-0.05 * * * \\
{[-3.77]}\end{array}$ & - & - & - & - & - & - & - & - \\
\hline Public Placement & - & - & - & $\begin{array}{l}-0.87 * * * \\
{[-2.90]}\end{array}$ & * & - & - & - & - & - & - \\
\hline Private Placement & & - & - & - & $\begin{array}{l}0.87 * * * \\
{[2.90]}\end{array}$ & - & - & - & - & - & - \\
\hline LnVol & - & - & - & - & - & $\begin{array}{l}0.48^{*} \\
{[1.56]}\end{array}$ & - & - & - & - & - \\
\hline DF & - & - & - & - & - & - & $\begin{array}{l}-0.45^{*} \\
{[-1.48]}\end{array}$ & - & - & - & - \\
\hline LC & - & - & - & - & - & - & - & $\begin{array}{l}0.02 * \\
{[1.27]}\end{array}$ & - & - & - \\
\hline CIR & - & - & - & - & - & - & - & - & $\begin{array}{l}-0.04 * * * \\
{[-2.94]}\end{array}$ & - & - \\
\hline$M / B$ & - & - & - & - & - & - & - & - & - & $\begin{array}{l}-0.002 * * * \\
{[-2.43]}\end{array}$ & - \\
\hline V_AR & - & - & - & - & - & - & - & - & - & - & $\begin{array}{l}-0.09 * * * \\
{[-3.60]}\end{array}$ \\
\hline$R^{2}$ & 0.10 & 0.06 & 0.36 & 0.10 & 0.10 & 0.21 & 0.09 & 0.08 & 0.48 & 0.05 & 0.10 \\
\hline $\bar{R}^{2}$ & 0.10 & 0.06 & 0.36 & 0.09 & 0.09 & 0.15 & 0.09 & 0.08 & 0.48 & 0.05 & 0.10 \\
\hline
\end{tabular}

Table 22: First 36-Months Price Performance Analysis Results (Dependent Variable - CAR)

\begin{tabular}{|c|c|c|c|c|c|c|c|c|c|c|c|}
\hline $\begin{array}{l}\text { Independent } \\
\text { Varible }\end{array}$ & Model 1 & Model 2 & Model 3 & 3 Model 4 & Model 5 & Model 6 & Model & 7Model \& & 8Model 9 & 9 Model 10 & OModel 11 \\
\hline LnOP & $\begin{array}{l}-3.29 * * \\
{[-6.68]}\end{array}$ & & - & - & - & - & - & - & - & - & - \\
\hline POF & - & $\begin{array}{l}-23.37 * * * \\
{[-18.25]}\end{array}$ & * & - & - & - & - & - & - & - & - \\
\hline LnASSET & - & - & $\begin{array}{l}-1.25^{* *} \\
{[-9.90]}\end{array}$ & * & - & - & - & - & - & - & - \\
\hline Public Placement & - & - & - & $\begin{array}{l}-17.97^{* * *} \\
{[-6.76]}\end{array}$ & * & - & - & - & - & - & - \\
\hline Private Placement & & - & - & - & $\begin{array}{l}17.97^{* * *} \\
{[6.76]}\end{array}$ & * & - & - & - & - & - \\
\hline LnVol & - & - & - & - & - & $\begin{array}{l}10.46^{* * * *} \\
{[6.19]}\end{array}$ & $*$ & - & - & - & - \\
\hline DF & - & - & - & - & - & - & $\begin{array}{l}-1.03 * * \\
{[-1.75]}\end{array}$ & - & - & - & - \\
\hline LC & - & - & - & - & - & - & - & $\begin{array}{l}0.77^{* * *} \\
{[3.82]}\end{array}$ & * & - & - \\
\hline CIR & - & - & - & - & - & - & - & - & $\begin{array}{l}-0.01 * * * \\
{[-6.95]}\end{array}$ & $*$ & - \\
\hline$M / B$ & - & - & - & - & - & - & - & - & - & $\begin{array}{l}-0.38 * * * \\
{[-3.92]}\end{array}$ & - \\
\hline V_CAR & - & - & - & - & - & - & - & - & - & - & $\begin{array}{l}-0.49 * * * \\
{[-10.88]}\end{array}$ \\
\hline$R^{2}$ & 0.09 & 0.17 & 0.03 & 0.09 & 0.09 & 0.76 & 0.19 & 0.16 & 0.18 & 0.16 & 0.37 \\
\hline $\bar{R}^{2}$ & 0.09 & 0.17 & 0.03 & 0.09 & 0.09 & 0.73 & 0.19 & 0.16 & 0.18 & 0.16 & 0.37 \\
\hline
\end{tabular}


Note: $*, * *$, and $* * *$ indicate that the relevant parameters are statistically significant at $10 \%, 5 \%$ and $1 \%$ significance level, respectively. Figures in the square brackets refer to $t$ statistics while figures in the normal brackets represent probability values.

\section{CONCLUSION}

In this study, the existence of long-term underperformance anomaly was investigated in Borsa Istanbul during the period of 2010-2015. For this purpose, the 3-year share price performance of companies that carried out seasoned equity offerings was analyzed in comparison with the market average return and peer groups' return. As a result of the analysis, the long-term underperformance anomaly was confirmed in Borsa Istanbul for the analysis period. Underperformance anomaly was observed during on basis of all years and sectors. It was determined that the return of investors who bought a share from the issuance and hold them during the 3-year period would be lower than the return of market average and peer group firms that match sample firms by assets size and M/B ratio. Abnormal returns that adjusted according to market and peer group average confirm underperformance anomaly in Borsa Istanbul for the analysis period.

\section{When long term price determinants are analyzed;}

In the analysis that abnormal return (AR) was used as the dependent variable, long-term price determinants were; The effects of private placement method, volume and firm leverage ratio are positive while offer price, offering frequency, asset size, public placement method, capital increase ratio, volatility, M/B ratio and the difference between obtained income and targeted income have a negative effect on the long-term share price performance.

In the analysis that cumulative abnormal return (CAR) was used as the dependent variable, long-term price determinants were;

The effects of private placement method, volume and firm leverage ratio are positive while offer price, offering frequency, asset size, public placement method, capital increase ratio, volatility, M/B ratio and the difference between obtained income and targeted income have a negative effect on the long-term share price performance.

The effect of the determinant factors on the long-term stock price performance of sample firms is summarized in Table 23 below.

\section{Table 23: Long Term Determinant Factors of Share Price Performance}

\begin{tabular}{lcc}
\hline $\begin{array}{l}\text { Independent Variable / } \\
\text { Period }\end{array}$ & AR CAR \\
\hline OP & - & - \\
POF & - & - \\
V & - & - \\
LnAsset & - & - \\
Public Placement & - & - \\
Private Placement & + & + \\
LnVol & + & + \\
DF & - & - \\
LC & + & + \\
CIR & - & - \\
MB & - & - \\
\hline
\end{tabular}

In the long run, it appears that the vast majority of the variables have a negative effect on stock price performance. It was determined that the remaining 8 variables have a long-term negative effect on the share price, except for the private placement method, transaction volume and leverage ratio. 


\section{REFERENCES}

Allen, D. E., Soucik, V. (2008). Long run underperformance of seasoned equity offerings fact or an illusion. Journal Mathematics and Computers in Simulation. 78 (2/3). $146-154$.

Asquith, P., Mullins, D. W. (1986). Equity issues and offering dilution. Journal of Financial Economics. 1 (15). 61 - 89.

Bessler, W., Thies, S. (2006). Initial public offerings, subsequent equity offerings and long - run performance: evidence from IPOs in Germany. The Journal of Entrepreneurial Finance, 1 (3). 1-37.

Bilinski, P., Liu, W., Strong, N. (2012). Does liquidity risk explain low firm performance following seasoned equity offerings. Journal of Banking and Finance. 36 (10). 2.770-2.785.

Chen, S. L., Lin, C. Y., Lin, Y. M. (2013). Ownership structure, idiosyncratic risk and financing policy. Journal of International Finance Studies. 13 (2). 51-62.

Clarke, J., Dunbar, C., Kahle, K. M. (2001). Long - run performance and insider trading in completed and canceled seasoned equity offerings. Journal of Financial \& Quantitative Analysis. 36 (4). 415-430.

Denis, D. J., Sarin, A. (2001). Is the market_surprised by poor earnings realizations following seasoned equity offerings?. The Journal of Financial and Quantitative Analysis. 36 (2). 169-193.

Dubois, M., Jeanneret, P. (2000). The long - run performance of seasoned equity offerings with rights. 1-37.

Eckbo, B. E., Masulis, R. W., Norli, Q. (2000). Seasoned public offerings: resolution of the new issues puzzle. Journal of Financial Economics. 56 (2). $251-291$.

Gao, X., Ritter, J. R. (2010). The marketing of seasoned equity offerings. The Journal of Financial Economics. 97 (1). 33 - 52.

Göçer, i. (2015). Ekonometri. İzmir : Lider Yayınları.

Gökkaya, S., Highfield, M. J. (2014). Sales of secondary shares In SEOs : a comparison across top managers, other insiders and outsiders. Financial Management Association International. 43 (4). 757-794.

Granger, C. W. J. (1969). Investigating causal relations by econometric models and cross-spectral methods. Journal of Econometric Society. (37). 424-438.

Gujarati, D.N. (2004). Basic econometrics (Fourth Edition): McGraw Hill.

Harjoto, M., Garen, J. (2003). Why do IPO firms conduct primary seasoned equity offerings?. The Financial Review. 38 (1). 103 - 125.

Harris, S. (2004). The long-term performance of UK stocks after making rights issues. Journal of Asset Management. 4 (5). 308 - 317.

He, Y., Wang, J., Wei, J. K. C. (2013). Information asymmetry before and after SEOs and SEO underpricing. Research Centre for International Economics Working Paper. City University of Hong Kong.

Huang, C. W., Ho, P. H., Lin, C., Yen, J. F. (2014). Firm age, idiosyncratic risk and long - run SEO underperformance. International Review of Economics \& Finance. (34). 246-266.

Huang, Z. (2012). Seasoned equity offerings in China. Phd Thesis, SOAS University of London.

Islam, M. S., Uddin, M. M., Ahmad, S. (2002). The operating performance of firms conducting seasoned equity offerings in Bangladesh. Journal of Business Studies. 23 (2). 1-42.

Jarnecic, E., Yubo, L. (2013). Equity issues and the impact of lead manager affiliation on broker market share and trading volume. Global Conference On Business \& Finance Proceedings. 1 (8).

Jeanneret, P. (2005). Use of the proceeds and long - term performance of French SEO firms. European Financial Management. 11 (1). 99 - 122.

Jegadeesh, N., Weinstein, M., Welch, I. (1993). An empirical investigation of IPO returns and subsequent equity offerings. Journal of Financial Economics. 34 (2). 153 - 175.

Jo, H., Kim, Y. (2008). Ethics and disclosure: a study of the financial performance of firms in the seasoned equity offerings market. Journal of Business Ethics. 80 (4). 855-878.

Jo, H., Kim, Y., Shin, D. (2012). Underwriter syndication and corporate governance. Review of Quantitative Finance \& Accounting. 38 (1). $61-86$.

Kim, D., Palia, D., Saunders, A. (2008). The impact of commercial banks on underwriting spreads: evidence from three decades. Journal of Financial \& Quantitative Analysis. 43 (4). 975-1000.

Loughran, T., Ritter, J. R. (1997). The operating performance of firms conducting seasoned equity offerings. Journal of Finance. 52 (5). 1823 1850.

Loughran, T. (1997). Book-to-market across firm size, exchange, and seasonality: is there an effect?. Journal of Financial and Quantitative Analysis. 32 (3). 249 - 268. 
Lyandres, E., Le, S., Lu, Z. (2008). The new issues puzzle: testing the investment-based explanation. Review of Financial Studies. 21 (6). $2825-$ 2855.

Ngatuni, P., Capstaff, J., Marshall, A. (2007). Long - term performance following rights issues and open offers in the UK. Journal of Business Finance and Accounting. 34 (1/2). 33 - 64.

Pedroni, P. (2004). Panel cointegration; asymptotic and finite sample properties of pooled time series tests with an application to the PPP hypothesis. Econometric Theory. 1(20) 597-625.

Pesaran, M. H. (2007). A simple panel unit root test in the presence of cross-section dependence. Journal of Applied Econometrics. 1(22). 265 312.

Ritter, J. R. (1991). The long - run performance of initial public offerings. Journal of Finance. 46 (1). 3 - 27.

Rubalcava, A. (2012). Valuation effects of the Sarbanes-Oxley act: evidence from seasoned equity offerings by Canadian cross-listed firms. Midwest Finance Association Annual Meeting Paper. 1-16.

Scholes, M. S. (1972). The market for securities: substitution versus price pressure and the effects of information on share prices. The Journal of Business. 45 (2). 179 - 211.

Seal, J. K., Matharu, J. S. (2012). Long run performance of initial public offerings and seasoned equity offerings in India. Working Paper, Indian Institute of Foreign Trade.

Spiess, D. K., Graves, J. A. (1995). Underperformance in long-run stock returns following seasoned equity offerings. Journal of Financial Economics. 38 (3). 243 - 267

Tarı, R. (2012). Ekonometri. Kocaeli : Umuttepe Yayınları.

Teoh, S. H., Welch, I., Wong, T. J. (1998). Earnings management and the underperformance of seasoned equity offerings. Journal of Financial Economics.1 (50). 63 - 99.

Virolainen, M. (2009). macro and micro determinants of seasoned equity offerings and issuer stock market performance. Master Thesis, Helsinki School of Economics Accounting And Finance Department.

Walker, M. D., Yost, K., Zhao, J. (2016). Credibility and multiple SEOs : what happens when firms return to the capital market?. Journal of Financial Management Association International. 45 (3). 67-70. 